\title{
Exosomal circRNAs: Emerging Players in Tumor Metastasis
}

\author{
Hao Zhou ${ }^{1}$, Xiaoyun $\mathrm{He}^{2}$, Yuxiang $\mathrm{He}^{3}$, Chunlin $\mathrm{Ou}^{4 *}$ and Pengfei Cao ${ }^{1,5 *}$ \\ ${ }^{1}$ Department of Hematology, Xiangya Hospital, Central South University, Changsha, China, ${ }^{2}$ Departments of Ultrasound Imaging, \\ Xiangya Hospital, Central South University, Changsha, China, ${ }^{3}$ Department of Oncology, Xiangya Hospital, Central South \\ University, Changsha, China, ${ }^{4}$ Department of Pathology, Xiangya Hospital, Central South University, Changsha, China, ${ }^{5}$ National \\ Clinical Research Center for Geriatric Disorders, Xiangya Hospital, Central South University, Changsha, China
}

OPEN ACCESS

Edited by:

César López-Camarillo, Universidad Autónoma de la Ciudad de México, Mexico

Reviewed by: Irina R. Matei, Cornell University, United States Nabanita Chatterjee, The Ohio State University, United States

Delphine Garnier, Sorbonne Université, France

${ }^{*}$ Correspondence: Pengfei Cao caopengfei66@163.com Chunlin Ou

ouchunlin@csu.edu.cn

Specialty section:

This article was submitted to Molecular and Cellular Oncology,

a section of the journal Frontiers in Cell and Developmental Biology

Received: 29 September 2021 Accepted: 18 November 2021 Published: 08 December 2021

Citation:

Zhou $\mathrm{H}, \mathrm{He} X, \mathrm{He} Y, \mathrm{Ou} \mathrm{C}$ and Cao $P$ (2021) Exosomal circRNAs: Emerging Players in Tumor Metastasis. Front. Cell Dev. Biol. 9:786224. doi: 10.3389/fcell.2021.786224
Metastasis is an important feature of malignant tumors, and is the primary cause of poor prognosis and treatment failure, in addition to representing a potentially fatal challenge for cancer patients. Exosomes are small extracellular vesicles $30-150 \mathrm{~nm}$ in diameter that transmit cargo, such as DNA, RNA, and proteins, as a means of intercellular communication. Exosomes play crucial roles in a range of human diseases, especially malignant tumors. A growing number of studies have verified that circRNAs can be enveloped in exosomes and transferred from secretory cells to recipient cells, thereby regulating tumor progression, especially tumor metastasis. Exosomal circRNAs regulate tumor cell metastasis not only by regulating the signaling pathways, but also by affecting the tumor microenvironment. Moreover, exosomal circRNAs have the potential to serve as valuable diagnostic biomarkers and novel therapeutic targets in cancer patients. In this review, we summarize the mechanism by which exosomal circRNAs modulate metastatic phenomena in various types of tumors, and put forward the prospects of clinical applications of exosomal circRNAs in tumor therapy.

Keywords: tumor metastasis, exosomes, circRNAs, tumor microenvironment, biomarkers, therapy

\section{INTRODUCTION}

Metastasis is one of the ten essential characteristics of malignant tumors and a hot research topic (Hanahan and Weinberg, 2011). It is a process that enables malignant cells to escape from the primary tumor site, migrate through the lymphatic and/or blood circulation, and ultimately spread to remote sites (Lazebnik, 2010; Tarin, 2011; Sun et al., 2015). The onset of tumor metastases is often indicative of poor prognosis, and $>90 \%$ of the cancer-related deaths result from metastases (Chambers et al., 2002; Maishi and Hida, 2017; Jiang et al., 2019; Mattiuzzi and Lippi, 2020; Wang et al., 2020d). Most metastatic lesions cannot be surgically eradicated because such lesion is often indicative of more widespread systemic disease (Gupta and Massagué, 2006). Although an increasing number or tumor treatments are being developed with advances in medical technology, tumor metastasis remains one of the major causes of the extremely high mortality rate in a variety cancers. Therefore, the search for tumor markers and therapeutic targets remains an important strategy for improved cancer treatment (Sun L. et al., 2019; Stoletov et al., 2020; Wang J. et al., 2020).

Exosomes are extracellular vesicles that originate from the multivesicular bodies (MVBs) and are present in intercellular space or circulate in biological fluids (Kalluri, 2016; Zhang and Yu, 2019; He et al., 2021a). These vesicles can be internalized by neighboring cells or by remote receptor cells through fusion with the target cell membrane, thereby altering the behavior of the target cell (Hessvik and Llorente, 2018; Gonda et al., 2019). Exosomes play integral roles in mediating intercellular 
communication, regulating immune system function (Gao et al., 2018; Yu et al., 2018), promoting cell development and differentiation (Zhou et al., 2021), influencing viral replication, and other physiological or pathological disease processes (Alenquer and Amorim, 2015) that affect the progression of many diseases, including tumors (Zhang and Yu, 2019). Recent studies have revealed that exosomes are involved in regulating several malignant biological behaviors of tumors by transporting various growth factors, proteins, lipids, nucleic acids, non-coding RNAs, and other molecules (Kalra et al., 2016), including promoting malignant proliferation, metastasis, and immune escape by tumor cells (Wortzel et al., 2019; Kugeratski and Kalluri, 2021), and contributing to tumor microenvironment (TME) (Meng et al., 2019; Wu et al., 2019). The roles played by non-coding RNAs (ncRNA)-a class of molecules present in high concentrations in exosomes, i.e., microRNAs (miRNAs), long non-coding RNAs (lncRNAs), and circular RNAs (circRNAs)-in tumor metastasis are gradually gaining attention (Zhao et al., 2015; Chen et al., 2020a; Guo et al., 2020). CircRNAs are covalently closed ncRNA molecules, comprising $3^{\prime}$ and $5^{\prime}$ ends joined in a non-collinear manner by reverse splicing (Zhang et al., 2016), which exhibit properties and functions different from those of linear RNA. Many studies have demonstrated that intracellular circRNAs can regulate tumor metastasis in multiple ways. However, the effect of exosomal circRNAs on tumor metastasis cannot be fully explained. Herein, we performed a systematic literature review of exosomal circRNAs in the context of development and progression of tumor metastases.

\section{TUMOR METASTASIS}

Metastasis of tumor cells is a multi-step process (He et al., 2021b) that includes the following steps: (I)tumor cells lose adhesion to neighboring cells and detach from the primary tumor, a phenomenon that results in the degradation of the extracellular matrix (ECM), and the migration and invasion of the cells into the surrounding tissues (Friedl and Wolf, 2003); (II) infiltration of the tumor cells into the bloodstream, followed by adaptation and escape from anoikis to survive in the circulation (Zhan et al., 2004), (III) exudation of the tumor cells outside the blood vessels (IV), and finally entry of the tumor cells into the metastatic site, followed by adaptation and growth, resulting in the eventual colonization of the site (Fidler, 2003; Nguyen et al., 2009; Scully et al., 2012) (Supplementary Figure S1). Epithelialto-mesenchymal transition (EMT) is an important form of tumor metastasis. EMT is a complex cellular pathway in which epithelial cells lose intercellular adhesion (characterized by loss of membrane E-Cad) and gain mesenchymal features (characterized by increased $\mathrm{N}$-cadherin expression and migration capacity) (Bakir et al., 2020). Stephen Paget first proposed the classic hypothesis of "seed and soil" in 1989. He compared the primary tumor to a "plant", the tumor cells to "seeds", and the host environment to "soil" (Paget, 1989), and boldly hypothesized, "When a plant has seeds, its seeds can be taken anywhere; but they can only survive and grow if they fall on suitable soil". This assumption is accepted as the fundamental theory for explaining tumors and metastasis (Langley and Fidler, 2011; Ribelles et al., 2014). In this doctrine, it is believed that the autonomous mechanisms of tumor cells are insufficient to accomplish metastasis and that tumor metastasis is regulated by other factors, including tumor microenvironment (TME) (Quail and Joyce, 2013; McAllister and Weinberg, 2014). Interactions between the TME and tumor tissue is gradually gaining attention as a new field (Quail and Joyce, 2013; Liao et al., 2021; Xiao and $\mathrm{Yu}, 2021)$. TME refers to the cellular environment in which the tumor exists, and its composition includes tumor cells as well as surrounding blood vessels, ECM, signaling molecules, and non-malignant cells such as fibroblasts and immune cells (Luo et al., 2016). Several studies have shown that the TME has an inhibitory effect on the growth of malignant tumors (Holmgren et al., 1995; Suzuki et al., 2006). However, in most malignant tumors, these inhibitions are overcome, resulting in the use of support cells by malignant tumors to increase their metastatic potential and promote their own growth and relocation to remote sites (Marx, 2013; Massagué and Obenauf, 2016). Tumor cells in highly aggressive primary tumors are more adept at exploiting this particular tissue microenvironment. Moreover, stromal cells and fibroblasts can also secrete growth factors such as hepatocyte growth factor (HGF), chemokines (e.g., CXCL12), and exosomes, which can promote the forming of pre-metastatic niche (PMN) (Filipazzi et al., 2012; Liu and Cao, 2016; Whiteside, 2016). These growth factors not only directly promote the growth and survival of malignant cells, but also act as decoys to stimulate other cells to migrate to the TME and indirectly promote tumor invasion and metastasis (Spaeth et al., 2008; Hanahan and Coussens, 2012). Metastasis is "a long journey" for the tumor cells themselves, as there are many rate-limiting steps in the formation of metastatic cancer, including extravasation, distal organ survival, and the establishment of sustained growth (Psaila and Lyden, 2009; Acharyya and Massague, 2016). Microenvironmental cues play important roles in all steps of metastasis. Thus, successful metastasis depends on the ability of cancer cells to adapt to different microenvironments at each step of the metastatic cascade (primary tumor, body circulation and final metastatic site) (Zhuang et al., 2019).

\section{BIOLOGICAL CHARACTERISTICS OF EXOSOMAL CIRCRNAS}

Exosomes are lipid bilayer nano-vesicles with a "spherical" morphology $(30-150 \mathrm{~nm})$ that are thought to be released by almost all cell types (Théry et al., 2002). These vesicles display a number of surface molecular markers, such as CD9, CD63, and CD81 (Mathivanan et al., 2010; Kowal et al., 2016). Exosome formation comprises four stages, i.e., initiation, endocytosis, formation of MVBs, and exosome secretion. In this process, the endosomal sorting complex required for transport (ESCRT) can select ubiquitin-tagged proteins, lead them to MVBs, and separate and release them from the peripheral membrane by a process similar to cytoplasmic division and 
viral outgrowth (Hanson and Cashikar, 2012; van Niel et al., 2018; $\mathrm{Xu}$ et al., 2018). Exosomes are widely available and have become essential mediators of intercellular communication in physiological and pathological states (Meldolesi, 2018; Delpech et al., 2019; Asghar et al., 2020). As a signal vector for intercellular communication, the content of exosomes varies with different physiological and pathological conditions and primary cell types (Bebelman et al., 2018). Many studies have reported that exosomes regulate tumor progression by carrying or delivering multiple biomodulator "cargoes" (including ncRNAs and proteins) (He et al., 2019; Li R. et al., 2019). Exosomes are key contributors to a wide range of biological processes during tumor growth and progression.

Exosomes contain a variety of RNA molecules, including mRNAs, miRNAs, lncRNAs, and circRNAs (Braicu et al., 2015; Yang and Li, 2018). Compared to other types of RNA, circRNAs are abundant and specific non-coding RNAs that are still not fully understood (Nie et al., 2020). CircRNAs was first discovered in Sendai virus and plant-like viruses by electron microscopy in 1976 (Kolakofsky, 1976). It has long been believed that these closed-loop covalent RNA molecules are a byproduct of rare error responses and have no specific functions (Cocquerelle et al., 1993). However, in recent years, many advances have been made in the study of circRNAs: circRNAs are produced by reverse splicing a 3' splice donor to an upstream 5' splice acceptor (Wilusz and Sharp, 2013). Because of this specific structure, circRNAs are resistant to exonucleases and exhibit greater stability than linear non-coding RNAs (such as miRNAs and lncRNAs) (Salzman et al., 2012; Jeck et al., 2013). CircRNA can be found in the cytoplasm, nucleus, or extracellular vesicles external to the cell. They usually perform different functions depending on their localization and distribution (Lasda and Parker, 2016; Ou et al., 2020). Although we have not been able to provide a complete explanation for the biological origins and functions of circRNAs, a large number of studies have confirmed that the main function of circRNAs is to effectively sponge miRNAs through a competitive endogenous RNA (ceRNA) mechanism, reduce their inhibitory effect on target genes (Hansen et al., 2013; Hu Y. et al., 2019; Liang et al., 2020), and activate or inhibit downstream signaling pathways by interacting with proteins (Du et al., 2017; Zang et al., 2020). Endogenous circRNAs have been reported to have the potential for translation (Legnini et al., 2017; Lei et al., 2020), and the products of translation may play important roles in disease progression.

CircRNAs can be encapsulated into exosomes that can be shared between cells. The entry of circRNAs into exosomes is influenced in part by the levels of relevant miRNAs in the parent cells (Li et al., 2015). The sorting of circRNAs species into exosomes may be positively regulated. This suggests that circRNAs are selectively encapsulated within exosomes (Hou et al., 2018). Interestingly, related studies have shown that circRNAs are much more enriched in exosomes than in the cells producing them (Dignat-George and Boulanger, 2011; Dou et al., 2016), and that exosomal circRNAs levels are only moderately correlated with the cellular circRNAs levels ( $\mathrm{Li}$ et al., 2015). Many questions still remain about the mechanisms involved in exosomal circRNAs enrichment. Emerging data indicate that exosomal circRNAs have multiple functions, such as promoting inflammatory responses, regulating hormone levels in the body, and modulating immunity (Table 1). For example, Wang et al. (Wang S. et al., 2020) found that circRNA-0077930 in exosomes released from endothelial cells induces vascular smooth muscle cell senescence in high-glucose environments. This study provides new insights into the mechanism of smooth muscle cell aging in a high-glucose environment. This finding has significant implications for the prevention of diabetic vascular disease and the exploration of new drug targets. Exosomes released from astrocytes deliver circSHOC2 (Chen et al., 2020b) to neuronal cells, thereby ameliorating ischemia-induced apoptosis and protecting neurons from ischemic injury. In case of hypoxia, circHIPK3 (Wang et al., 2020e) is transferred to myocardial endothelial cells via exosomes released from cardiomyocytes. CircHIPK3mediated VEGFA overexpression significantly increases cell proliferation and migration, which preserves the function and integrity of post-infarction myocardial endothelial cells and exhibits cardioprotective effects.

Recently, Li et al. (Li et al., 2015) first revealed the presence and enrichment of circRNAs in cancer-derived exosomes by RNA-seq analysis. CircRNAs play significant roles in regulating tumorigenesis, tumor cell progression, metastasis, and drug resistance development. For instance, Xiao et al. (Dai et al., 2018) transferred circRNA_100284 secreted by arsenitetransformed human liver epithelial cells (L-02) to normal L-02 cells via exosomes. CircRNA_100284 acted as a sponge for miRNA-217 to accelerate the cell cycle and promote cell proliferation, thereby inducing malignant transformation of L02 cells. This mechanism provides a new explanation for arseniteinduced cancer. Glioma-derived exosomal circRNA-0001445 (Han et al., 2021) significantly promotes proliferation and inhibits the apoptosis of glioma cells via the miRNA-1275p/SNX5 signaling pathway. Shi et al. (Xiao and Shi, 2020) found that exosomal circRNA_400068 produced by renal cell carcinoma cells promotes the proliferation of healthy kidney cells, significantly inhibits their apoptosis, and promotes their transformation to malignancy, which may be due to signaling through regulation of the miRNA-210-5p/SocS1 axis pathway. Circ_UBE2D2 was found to be upregulated in exosomes isolated from triamcinoloneresistant breast cancer cells (Hu et al., 2020). Exosomes enhance the resistance of breast cancer cells to triamcinolone by mediating the transfer of circRNA_UBE2D2 into non-triamcinoloneresistant breast cancer cells. This mechanism may suggest the prospect of a promising candidate biomarker and therapeutic target for drug resistance in breast cancer.

\section{FUNCTIONS AND MECHANISMS OF EXOSOMAL CIRCRNA IN TUMOR METASTASIS}

Interestingly, researchers have identified that tumor cells produce more than 10 times more exosomes than normal cells. The enrichment and stable presence of circRNAs in exosomes and 
TABLE 1 | Role of exosomal circRNAs in human diseases.

\begin{tabular}{|c|c|c|c|c|c|}
\hline Diseases & $\begin{array}{l}\text { Exosomal } \\
\text { circRNA }\end{array}$ & Secreting cells & Recipient cells & Effect & Ref. \\
\hline Diabetic retinopathy & $\begin{array}{l}\text { CircRNA- } \\
\text { cPWWP2A }\end{array}$ & Vascular epidural cells & Endotropical cells & $\begin{array}{l}\text { Involved in diabetes-induced retinal } \\
\text { vascular dysfunction }\end{array}$ & $\begin{array}{l}\text { Liu et al. } \\
\text { (2019) }\end{array}$ \\
\hline $\begin{array}{l}\text { Diabetic vascular } \\
\text { disease }\end{array}$ & CircRNA-0077930 & Endothelial cells & Vascular smooth muscle cells & $\begin{array}{l}\text { Induced vascular smooth muscle cell } \\
\text { senescence }\end{array}$ & $\begin{array}{l}\text { Wang et al. } \\
\text { (2020c) }\end{array}$ \\
\hline $\begin{array}{l}\text { Polycystic ovary } \\
\text { syndrome }\end{array}$ & CircLDLR & KGN cells & KGN cells & Regulation of estrogen secretion & $\begin{array}{l}\text { Huang et al. } \\
\text { (2020b) }\end{array}$ \\
\hline Myocardial infarction & CircHIPK3 & Cardiomyocytes & Cardiac endothelial cells & $\begin{array}{l}\text { Promotes angiogenesis at the border } \\
\text { around the infarcted area }\end{array}$ & $\begin{array}{l}\text { Wang et al. } \\
\text { (2020e) }\end{array}$ \\
\hline Ischemic stroke & CircSHOC2 & Astrocyte & Neuronal cells & $\begin{array}{l}\text { Inhibits neuronal apoptosis and ameliorates } \\
\text { neuronal damage }\end{array}$ & $\begin{array}{l}\text { Chen et al. } \\
\text { (2020b) }\end{array}$ \\
\hline $\begin{array}{l}\text { Intervertebral disc } \\
\text { degeneration }\end{array}$ & CircRNA_0000253 & $\begin{array}{l}\text { Degenerative nucleus } \\
\text { pulposus cells }\end{array}$ & $\begin{array}{l}\text { Normal nucleus pulposus } \\
\text { cells }\end{array}$ & $\begin{array}{l}\text { Promotes apoptosis and inhibits } \\
\text { proliferation of Normal nucleus pulposus } \\
\text { cells }\end{array}$ & $\begin{array}{l}\text { Song et al. } \\
(2020)\end{array}$ \\
\hline Osteoarthritis & CircRNA3503 & $\begin{array}{l}\text { Synovium mesenchymal } \\
\text { stem cells }\end{array}$ & Chondrocyte & $\begin{array}{l}\text { Promotes chondrocyte proliferation and } \\
\text { migration }\end{array}$ & $\begin{array}{l}\text { Tao et al. } \\
(2021)\end{array}$ \\
\hline Breast cancer & Circ_UBE2D2 & $\begin{array}{l}\text { Triamcinolone-resistant } \\
\text { breast cancer cells }\end{array}$ & $\begin{array}{l}\text { Non-triamcinolone-resistant } \\
\text { breast cancer cells }\end{array}$ & $\begin{array}{l}\text { Enhance the resistance of breast cancer } \\
\text { cells to triamcinolone }\end{array}$ & $\begin{array}{l}\text { Hu et al. } \\
(2020)\end{array}$ \\
\hline Glioma & CircRNA-0001445 & Glioma cells & Glioma cells & $\begin{array}{l}\text { Promotes proliferation and inhibits the } \\
\text { apoptosis of glioma cells }\end{array}$ & $\begin{array}{l}\text { Han et al. } \\
(2021)\end{array}$ \\
\hline
\end{tabular}

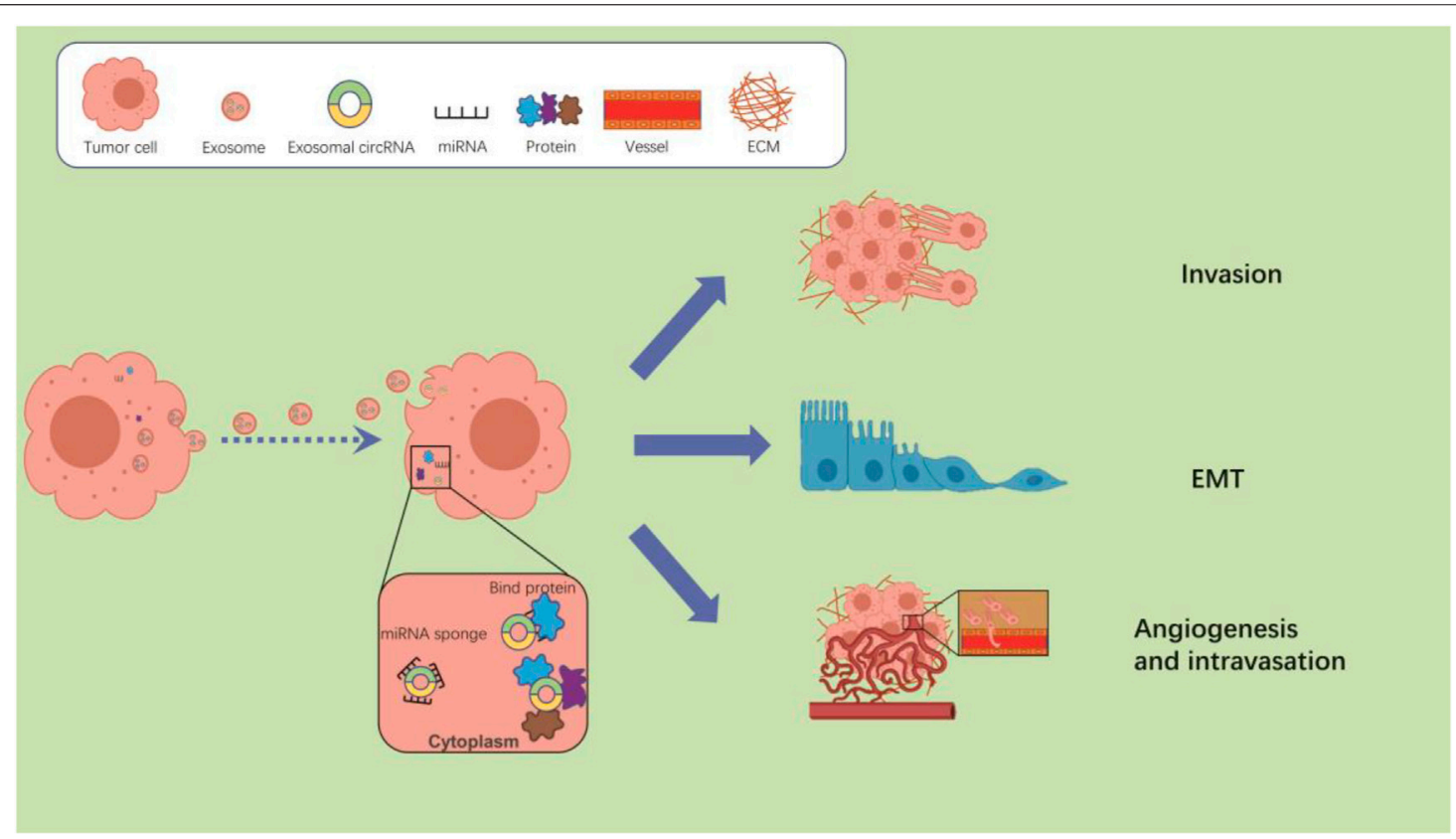

FIGURE 1 | The potential mechanisms of exosomal circRNAs regulate tumor metastasis. After taken up by cancer cells, exosomal circRNAs can regulate the tumor metastasis by interacting with miRNAs or proteins.

their intercellular transmission strongly implicate them in tumor metastasis (Li et al., 2015; Bao et al., 2016; Bai et al., 2019). There is increasing evidence that exosomal circRNAs play a key role in the metastasis of various cancers. Exosomal circRNAs may regulate tumor metastasis through a variety of different mechanisms, 1) cytoplasmic circRNAs adsorb miRNAs by sponging and de-repress miRNA-regulated genes, 2) circRNAs delivered to recipient cells can act as a protein sponge or decoy by adsorbing one or more proteins through specific binding sites, 3) exosomal circRNAs are able to regulate the TME through interactions with the immune system cells (Figure 1).

Figure 1 The potential mechanisms of exosomal circRNAs regulate tumor metastasis. After taken up by cancer cells, exosomal circRNAs can regulate the tumor metastasis by interacting with miRNAs or proteins. Exosomal circRNAs regulate tumor metastasis by sponging miRNAs. 
CircRNAs represent upstream regulatory molecules with a large number of miRNA response elements (MREs) (Memczak et al., 2013; Lasda and Parker, 2014). CircRNAs can be delivered to recipient cells via exosomes and bind to miRNAs, acting as an "miRNA sponge" by blocking the binding of miRNAs to target gene 3'- untranslated regions (UTRs) to disable miRNAs and restore the translation of proteins inhibited by specific miRNAmRNA binding events, thereby regulating tumor metastasis (Zhong et al., 2018; Verduci et al., 2019). CircRNAs exhibit more preferential binding to miRNAs than other endogenous RNAs (e.g., lncRNAs), hence the name (Shi et al., 2020) "super sponge". Xie et al. (Xie et al., 2020) found that exosome circSHKBP1 increased HUR expression in gastric cancer (GC) tissues through sponging of miR-582-3p. HUR is reported to be part of the VEGF signaling pathway that promotes VEGF secretion and induces angiogenesis, which promotes GC metastasis. In triple-negative breast cancer (TNBC) (Yang S.-j. et al., 2021, 1), breast cancer cell metastasis is promoted through the direct targeting of AKT1 via the exosome circPSMA1 that functions as a sponge for miR-637, which activates the AKT1/ $\beta$-catenin signaling pathway to regulate cell proliferation and migration. High expression of AKT1 and low expression of mir637 are highly correlated with poor prognosis in TNBC patients with lymph node metastasis. CircPSMA1 overexpression significantly enhances the metastatic capacity in the liver and lungs of mice. Zeng et al. (Zeng et al., 2020) found that overexpression of circFNDC3B severely inhibits angiogenesis in a mouse model of colorectal cancer (CRC). Treatment with exosomal circFNDC3B inhibits CRC cell growth, angiogenesis, and liver metastasis in vivo. Liu et al. (Liu et al., 2020) found that circ_MMP2 could be delivered to less invasive hepatocellular carcinoma (HCC) cells via exosomes derived from highly invasive HCC cells. Circ_MMP2 can upregulate the expression of its host gene matrix metallopeptidase $2(M M P 2)$ by acting as a sponge for miR-136-5p, a metastasis-associated RNA that promotes HCC cell metastasis.

EMT is one of the initiating steps of primary tumor invasion (Nishiyama et al., 2018; Shang et al., 2019). Exosomal circRNAs can promote tumor metastasis by sponging miRNAs, thereby promoting the EMT phenotype. For example, circPRMT5 (Chen et al., 2018) is expressed at abnormally high levels in urothelial carcinoma of the bladder (UCB) cells, and can be secreted into the blood and urine via exosomes. High levels of exosomal circPRMT5 in the serum and urine are positively correlated with lymph node metastasis and tumor progression. CircPRMT5 contributes to a significant reduction in SNAIL1 downregulation by reducing the inhibitory effect of miR-30c, which in turn promotes UCB cell invasiveness and EMT. Statistical analyses showed that high expression of circPRMT5 in UCB tissues is positively correlated with late $\mathrm{T}$ and $\mathrm{N}$ stages, and is associated with low disease-free survival (DFS). CircNRIP1 is a promoter of EMT in GC (Zhang et al., 2019). The exosomal circNRIP1 sponges miR-149-5p to regulate the expression of AKT1 in GC, which in turn exerts a tumor-promoting effect in the context of EMT. Circ_PVT1 (Wang H. et al., 2020) can enter exosomes originating in cervical cancer cells and function as a tumor promoter by inducing EMT in cervical cancer cells by targeting miR-1286, which in turn promotes tumor metastasis (Table 2).

RNA binding proteins (RBPs), a class of proteins involved in gene transcription and translation, are essential elements of circRNA function. Bioinformatic analysis of circRNA sequences reveals a low enrichment of RBP-binding sites compared to the corresponding linear RNAs. However, the unique tertiary structure of circRNA leads to a greater protein binding capacity than linear RNA sequences, allowing better interaction with proteins (Hentze and Preiss, 2013; You et al., 2015; Liang et al., 2018; Huang A. et al., 2020). CircRNAs can function as protein sponges (Yang et al., 2017), decoys (Abdelmohsen et al., 2017), scaffolds, or recruiters (Zeng et al., 2017; Sun Y.-M. et al., 2019) in different physiological and pathological environments. CircRNA-protein interactions also play pivotal roles in the regulation of tumor metastasis.

In HCC, the exosomal circ-0004277 (Zhu et al., 2021) derived from HCC cells blocks the binding of HuR to ZO-1 mRNA by competitive interactions with $\mathrm{HuR}$ protein, which in turn stimulates EMT progression by inhibiting ZO-1. Moreover, exosome-delivered circ-0004277 induces EMT in adjacent normal cells, further promoting the invasion of HCC cells into the surrounding normal tissues. Similarly, Xie et al. (Xie et al., 2020) found that exosomal circSHKBP1 could not only promote GC progression by regulating the miR-582-3p/HUR/VEGF pathway, but also promotes tumor growth and lung metastasis by sequestering HSP90 away from STUB1. Xu et al. (Xu Y. et al., 2021) explored the function of circ-CCAC1 in cholangiocarcinoma (CAA), and found that circ-CCAC1 could translocate into vascular endothelial cells via exosomes and bind to $\mathrm{EZH} 2$ in the cytoplasm to inhibit the expression of intercellular junctional proteins (ZO-1 and occludin) that control endothelial cell permeability. Decreased expression of intercellular linker proteins disrupts the vascular endothelial barrier and induces angiogenesis, thereby promoting the formation of pre-metastatic ecological niches and providing a supportive microenvironment for the spread of cancer cells.

\section{EXOSOMAL CIRCRNA INTERACTS WITH THE TUMOR MICROENVIRONMENT TO REGULATE TUMOR METASTASIS}

The TME is complicated and ever-evolving. In addition to stromal cells, fibroblasts, and endothelial cells, the TME includes both innate and adaptive immune cells (Hinshaw and Shevde, 2019). Adaptive immune cells are mainly T lymphocytes, which can directly contribute to, or stimulate other cells in the TME to influence tumor growth. They can be classified as "Th1" and "Th2" cells based on their differentiation status. Th1 cells control the pro-inflammatory phenotype, and Th2 cells coordinate the immunosuppressive phenotype (McGuirk and Mills, 2002). The innate immune cell types include macrophages, dendritic cells (DCs), neutrophils, myeloidderived suppressor cells (MDSCs), natural killer cells (NK), and innate lymphocytes (ILC). The innate immune response can have a significant impact on the TME (Joyce and Pollard, 
TABLE 2 | Regulatory model of "Exosomal circRNA-miRNAs-mRNA" in tumor metastasis.

\begin{tabular}{|c|c|c|c|c|c|}
\hline Tumor types & Exosomal circRNA & miRNA & Targeted gene & Signaling pathways & Ref. \\
\hline \multirow[t]{2}{*}{ GC } & CircSHKBP1 & miR-582-3p & HUR & VEGF signaling pathway & Xie et al. (2020) \\
\hline & CircNRIP1 & miR-149-5p & AKT1 & mTOR pathway & Zhang et al. (2019) \\
\hline \multirow[t]{3}{*}{$\mathrm{HCC}$} & Circ-ZNF652 & miR-29a-3p & GUCD1 & - & (Li et al. (2020b) \\
\hline & CircPTGR1 & miR-449a & MET & - & Wang et al. (2019a) \\
\hline & Circ_MMP2 & miR-136-5p & MMP2 & - & Liu et al. (2020) \\
\hline \multirow[t]{3}{*}{ NSCLC } & Circ_100395 & $\operatorname{miR}-141-3 p$ & LATS2 & Hippo/YAP signaling pathway & Zhang et al. (2021) \\
\hline & CircSETDB1 & $\mathrm{miR}-7$ & sp1 & - & Xu et al. (2021a) \\
\hline & CircARHGAP10 & miR-638 & FAM83F & - & Jin et al. (2019) \\
\hline \multirow[t]{4}{*}{ CRC } & CircPACRGL & miR-142-3p/miR-506-3p & TGF- $\beta 1$ & - & Shang et al. (2020) \\
\hline & CircFNDC3B & $\operatorname{miR}-937-5 p$ & TIMP3 & VEGF signaling pathway & Zeng et al. (2020) \\
\hline & CirclFT80 & miRNA-1236-3p & HOXB7 & - & Feng et al. (2019) \\
\hline & Circ-133 & miR-133a & GEF-H1/RhoA & - & Yang et al. (2020) \\
\hline Ovarian Cancer & CircRNA051239 & miR-509-5p & PRSS3 & - & Ma et al. (2021) \\
\hline Prostate Cancer & Circ_0044516 & miR-29a-3p & - & - & Li et al. (2020a) \\
\hline Triple-negative breast cancer & CircPSMA1 & miR-63 & AKT1 & AKT1/ $\beta$-catenin signaling pathway & Yang et al. (2021b) \\
\hline Esophageal Cancer & Circ-048117 & $\operatorname{miR}-140$ & TLR4 & - & Lu et al. (2020) \\
\hline Cervical Cancer & Circ-PVT1 & miR-1286 & - & - & Wang et al. (2020a) \\
\hline \multirow[t]{2}{*}{ Pancreatic Cancer } & Circ-IARS & miR-122 & RhoA & - & Li (2018) \\
\hline & CircPED8A & miRNA-338 & MACC1 & MET/AKT and ERK pathway & Li et al. (2018b) \\
\hline Laryngeal squamous cell carcinoma & CircRASSF2 & miRNA-302b-3p & IGF-1R & - & Linli et al. (2019) \\
\hline
\end{tabular}

Exosomal circRNAs regulate tumor metastasis by binding proteins.

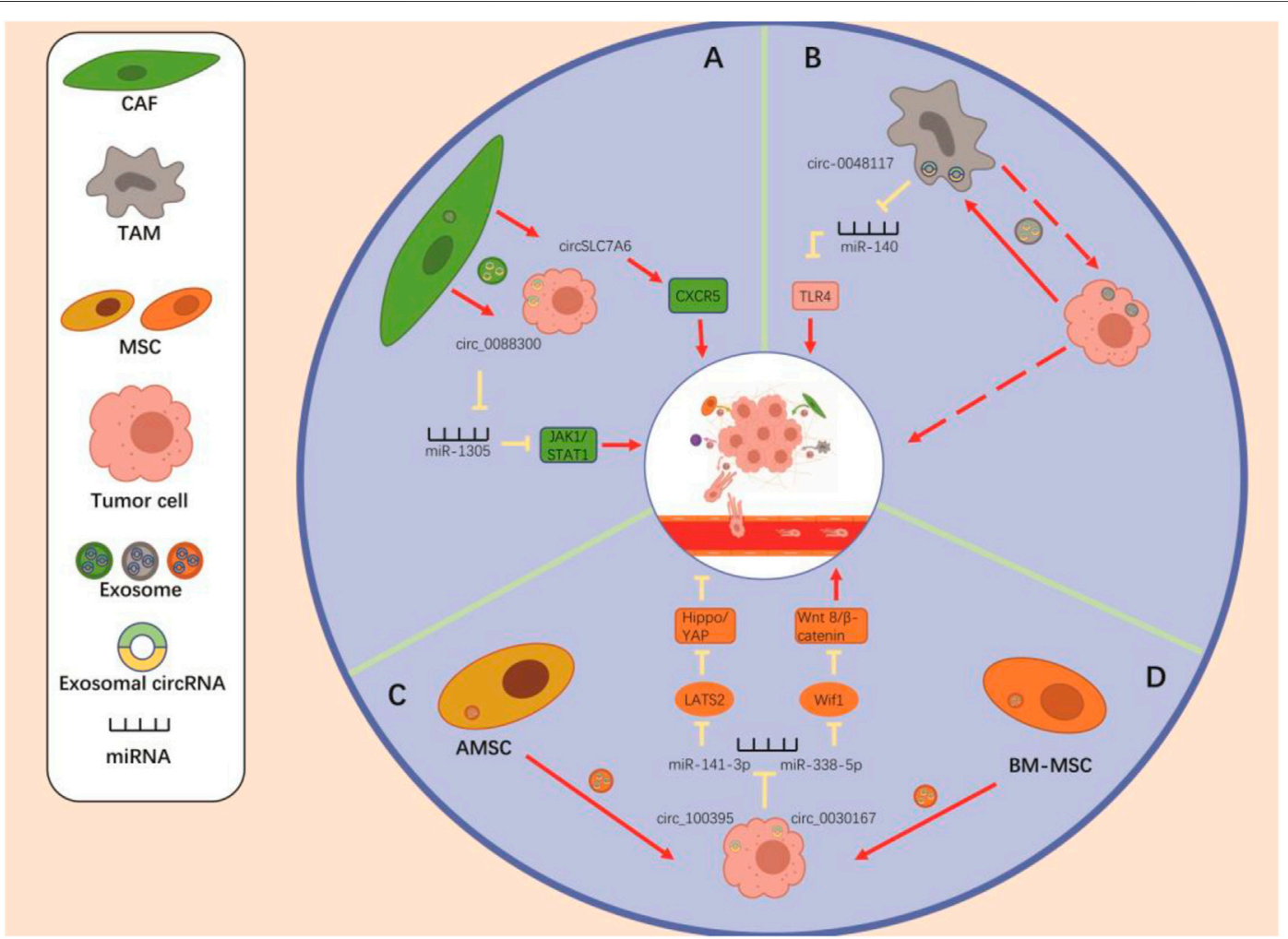

FIGURE 2 | The crosstalk "Non-tumor cells to tumor cells" via the exosomal circRNAs to regulate the tumor metastasis. (A) CAF-derived exo-circ_0088300 and exo-circSLC7A6 promote the tumor metastasis through regulating the miR-1305/JAK1/STAT1 axis and the expression of CXCR5. (B) Tumor-derived exosomal circ0048117 promotes the polarization of M2 macrophages by regulating the miR-140/TLR4 axis, thereby enhancing the metastatic potential of tumor cells. (C) AMSCderived exosomal circ_100395 inhibits the tumor metastasis via regulating the miR-141-3p/LATS2/Hippo/YAP pathway. (D) BM-MSC-derived exosomal circ_0030167 promotes tumor metastasis by regulating the miR-338-5p/Wif1/WNT8/ $\beta$-catenin axis in tumor cells. Regulation of mesenchymal stem cells promotes tumor metastasis. 
2009) either directly or indirectly (through control of T-cell fate) (Hinshaw and Shevde, 2019). In addition, immune cells interact with stromal cells, thus influencing tumor development (Tlsty and Coussens, 2006). Here, we focus on mesenchymal stem cells (MSCs), macrophages, and tumor-associated fibroblasts (Figure 2).

\section{Regulation of MSCs Promotes Tumor Metastasis}

MSCs are cells with multiple differentiation capabilities that can differentiate into osteoblasts, chondrocytes, adipocytes, and other cells of mesenchymal origin. It is well known that MSCs are recruited to tumor site thanks to the secretion of soluble factors. Based on this phenomenon, MSCs were explored to determine whether they could regulate nearby cancer cells at the primary site (Ridge et al., 2017). MSCs can produce exosomes, which may deliver signaling molecules by acting as paracrine mediators. Therefore, multiple cellular pathways are controlled to regulate tumor cell proliferation, angiogenesis, and metastasis (Zhao et al., 2020). Currently, whether MSC-derived exosomes promote or suppress tumors remains controversial, depending on the source of exosomes AND the model/tumor cells used as recipient for these exosomes (Vakhshiteh et al., 2019).

In recent years, exosomes released by MSCs have emerged as key regulators of tumor progression in various malignancies. Yao et al. (Yao et al., 2021) found that bone marrow MSC (BM-MSC)derived exosome circ_0030167 enhances Wif1 expression through regulation of miR-338-5p, which in turn inhibits the Wnt $8 / \beta$-catenin pathway, promoting invasion, migration, and proliferation, as well as tumor stemness in pancreatic cancer cells. Its emergence thus offers a new perspective for the treatment of pancreatic cancer. Interestingly, exosomal circ_100395 from adipose-derived MSC (AMSC) (Zhang et al., 2021) adsorbs miR-141-3p to increase the expression of LATS2, a protein that inhibits tumor cell proliferation, promotes apoptosis, and induces YAP phosphorylation, thereby reducing Hippo pathway activity. The YAP/Hippo pathway promotes EMT and progression of non-small cell lung cancer (NSCLC) cells. Therefore, exosomal circ_100395 inhibits progression and metastasis through the Hippo/YAP signaling pathway that regulates the miR-141-3p/LATS2 axis.

\section{Regulation of Tumor-Associated Macrophages Promote Tumor Metastasis}

Macrophages are among the most plastic, versatile cells of the body. However, these characteristics may be exploited by tumors to trigger different functions at different stages of tumor development (Qian and Pollard, 2010). Macrophages present in tumors are commonly referred to as tumor-associated macrophages (TAMs) and exhibit two distinct polarization phenotypes: Classical activation type (M1) and alternative activation type (M2). M1 macrophages promote the early stages of tumorigenesis by creating an inflammatory microenvironment (Mantovani and Sica, 2010). During tumor progression, TAMs undergo a phenotypic switch to the M2 phenotype. TAM cell populations composed of M2 macrophages play roles in clearing cellular debris, enhancing angiogenesis, and promoting tumor invasion and metastasis (Biswas et al., 2008; Maniecki et al., 2012).

Through both in vivo and in vitro experiments, Wang et al. (Wang et al., 2021) demonstrated that hsa_circ_00074854 secreted by HCC cells can be delivered to macrophages via exosomes, inducing macrophage M2 polarization, thereby promoting migration, invasion, and metastasis of HCC cells. In lung cancer, Katopodi et al. (Katopodi et al., 2021) found that upregulation of exosomal circHIPK3/PTK2 expression promotes the differentiation of monocytes into CD163 + M2 macrophages, which may play an important role in directing lymph node metastasis. In addition, Lu et al. (Lu et al., 2020) found that esophageal squamous cell carcinoma cells in a hypoxic microenvironment produced hsa-circ-0048117-rich exosomes, which promote the polarization of M2 macrophages and enhance the invasiveness and metastatic ability of tumor cells. Other researchers have suggested that macrophages represent a part of the malignant cell population in human tumors. Furthermore, studies have confirmed that TAMs also secrete exosomes, and whether TAMs can influence tumor metastasis through exosomal circRNAs is a direction worthy of future investigations. In conclusion, the roles of macrophages in metastasis require further investigation.

\section{Regulation of Tumor-Associated Fibroblasts Promotes Tumor Metastasis}

Fibroblasts are a major multifunctional cell type in connective tissue that deposit ECM and basement membrane components, regulate related epithelial differentiation events and immune responses, and mediate homeostasis (Li Y.-Y. et al., 2018). Cancer-associated fibroblasts (CAFs) are important components of the TME. CAFs are a major source of secretory growth factors, such as VEGF and pro-inflammatory factors, that are thought to contribute to tumor proliferation, invasion, and metastasis (Kato et al., 2018; Fan et al., 2020).

CAFs have been shown to deliver functional circ_0088300 (Shi et al., 2021) to GC cells via exosomes, thereby promoting the proliferation, migration, and invasive capacity of such cells. The inhibition of exosomal circ_0088300 may represent a new therapeutic strategy for GC. In addition, CAF-derived exosomes also promote metastasis and invasion of CRC ( $\mathrm{Hu}$ J. L. et al., 2019; Gu et al., 2020), breast cancer (Chen et al., 2021) and endometrial cancer cells (Bl et al., 2019), but the roles of exosomal circRNAs remain to be explored.

\section{EXOSOMAL CIRCRNAS AS NOVEL BIOMARKERS AND TARGETS OF TUMOR METASTASIS Exosomal CircRNA may Serve as Novel Biomarkers of Tumors Metastasis}

Exosomes have been found to be widely present in various body fluids, including saliva, plasma, urine, breast milk, amniotic fluid, 
TABLE 3 | Exosomal circRNA serve as novel biomarkers of tumor metastasis.

\begin{tabular}{|c|c|c|c|c|c|c|}
\hline Tumor types & $\begin{array}{l}\text { Exosomal } \\
\text { CircRNAs }\end{array}$ & $\begin{array}{l}\text { Sample } \\
\text { types }\end{array}$ & Expression & $\begin{array}{c}\text { Relationship to } \\
\text { clinicopathological features }\end{array}$ & AUC & Ref. \\
\hline \multirow[t]{4}{*}{ NSCLC } & CircSATB2 & Serum & Upregulated & Distant metastasis & 0.797 & Zhang et al. (2020) \\
\hline & Circ-0056616 & Plasma & Downregulated & TNM Stages & 0.812 & He et al. (2020) \\
\hline & CicHIPK3/PTK2 & Serum & Upregulated & Lymph node metastasis & - & $\begin{array}{l}\text { Katopodi et al. } \\
\text { (2021) }\end{array}$ \\
\hline & CircRNA-102481 & Serum & Upregulated & TNM Stages; Brain metastasis & - & Yang et al. (2021a) \\
\hline SCLC & Exo-FECR1 & Serum & Upregulated & Lymph node metastasis; Stages & - & Li et al. (2019a) \\
\hline Nasopharyngeal Carcinoma & CircMYC & Serum & Upregulated & TNM Stages & - & Luo et al. (2020b) \\
\hline $\begin{array}{l}\text { Oral squamous cell } \\
\text { carcinoma }\end{array}$ & Circ_0000199 & Serum & Upregulated & TNM Stages & - & Luo et al. (2020a) \\
\hline Prostate Cancer & Circ_0044516 & Serum & Upregulated & - & - & Li et al. (2020) \\
\hline \multirow[t]{2}{*}{ Pancreatic Cancer } & Circ-PDE8A & Plasma & Upregulated & $\begin{array}{l}\text { Duodenal infiltration; Vascular invasion; TNM } \\
\text { Stages }\end{array}$ & - & Li et al. (2018b) \\
\hline & Circ-IARS & & Upregulated & Liver metastasis; TNM Stages & - & Li, (2018) \\
\hline \multirow[t]{2}{*}{ Esophageal Cancer } & Circ_0026611 & Serum & Upregulated & Lymph node metastasis & 0.724 & Liu et al. (2021) \\
\hline & Circ-0048117 & & Upregulated & TNM Stages & - & Lu et al. (2020) \\
\hline $\mathrm{HCC}$ & Circ-100338 & Serum & Upregulated & $\begin{array}{l}\text { TNM Stages; Vascular invasion; Pulmonary } \\
\text { metastasis }\end{array}$ & - & $\begin{array}{l}\text { Huang et al. } \\
\text { (2020c) }\end{array}$ \\
\hline UCB & CircPRMT5 & Serum/Urine & Upregulated & Lymph node metastasis & - & Chen et al. (2018) \\
\hline CRC & Circ-0004771 & Serum & Upregulated & TNM Stages & 0.88 & Pan et al. (2019) \\
\hline \multirow[t]{3}{*}{ Cholangiocarcinoma } & Circ_0000284 & Plasma & Upregulated & TNM Stages & - & Wang et al. (2019b) \\
\hline & Circ-CCAC1 & Serum & Upregulated & TNM Stages & 0.759 & Xu et al. (2021b) \\
\hline & & Bile & & & 0.857 & \\
\hline
\end{tabular}

Exosomal circRNAs as targets of tumors cell metastasis.

and bile, which is very convenient for non-invasive testing (Kim et al., 2007). CircRNAs are conserved, stable, and cell- and tissuespecific (Perez de Acha et al., 2020). All of these properties provide strong support for exosomal circRNAs as candidate molecular diagnostic and therapeutic prognostic markers, showing good promise for application as molecular markers in non-invasive tests. Many studies have shown that variable expression of exosomal circRNAs in body fluids is associated with tumor metastasis. Zhang et al. (Zhang et al., 2020) reported that the expression of exosomal circSATB2 is associated with lymphatic metastasis in lung cancer. The expression of circSATB2 was higher in exosomes derived from sera of patients with metastatic lung cancer than in non-metastatic lung cancer, and the receiver operating characteristic (ROC) curve analysis showed its high sensitivity and specificity as a blood test for the diagnosis of lung cancer and lung cancer metastasis. It has also been reported that the expression of exosomal hsa_circRNA_0056616 (He et al., 2020) is significantly lower in patients with lymph node metastasis in lung adenocarcinoma than in patients without lymph node metastasis, and its expression is correlated with tumor-lymph node metastasis (TNM) staging. Plasma exosomal hsa_circRNA_0056616 may be a potential biomarker for predicting lymph node metastasis in lung adenocarcinoma, and its expression level may be a valuable biomarker for the treatment of lymph node metastasis in lung adenocarcinoma. There are many similar studies in other cancer models, and here we list the most representative ones (Table 3). Circ-IARS is abundant in plasma exosomes of patients with metastatic pancreatic cancer (Li et al., 2018). Its high expression is associated with tumor vascular infiltration, liver metastasis, and TNM stage. Similarly, Wu et al. (Li Z. et al., 2018) found that high expression of plasma exosomal circ-PDE8A is associated with duodenal and vascular infiltration, or tumor TNM staging in pancreatic ductal adenocarcinoma, which is correlated with tumor progression and prognosis. Exosomal circ-PDE8A may be a useful marker of pancreatic ductal adenocarcinoma progression. In addition, $\mathrm{Li}$ et al. (Li et al., 2020) analyzed the expression of circ_0044516 in patients with high or low levels of metastases in prostate cancer. They found that circ_0044516 levels were higher in blood exosomes of highly metastatic prostate cancer cases than in blood exosomes of low metastatic prostate cancer patients. This finding indicates that exosomal circ_0044516 may be used as a serum marker to measure prostate cancer metastasis, opening a new direction for the treatment of this malignancy. Interestingly, exosomes levels are not only meaningful when measured in blood, but also have value when detected in other body fluids. In urinary exosomes from patients with UCB (Chen et al., 2018), the expression of circPRMT5 is substantially increased compared to that in healthy controls, and its expression level is also associated with lymph node metastasis and tumor progression. Although our research surrounding exosomal circRNAs is still in its infancy, many studies have demonstrated the potential of circRNAs as markers of cancer.

\section{Exosomal CircRNAs as Targets of Tumors Metastasis}

Many tumor metastasis-associated exosomal circRNAs have potential clinical applications (Figure 3). By comparing mice injected with circFNDC3B-containing exosomes and normal control exosomes, Zeng et al. (Zeng et al., 2020) found that mice separately injected with exosomal circFNDC3B had reduced tumor volume and weight, and a substantial reduction in VEGFR expression relative to mice injected with normal control 


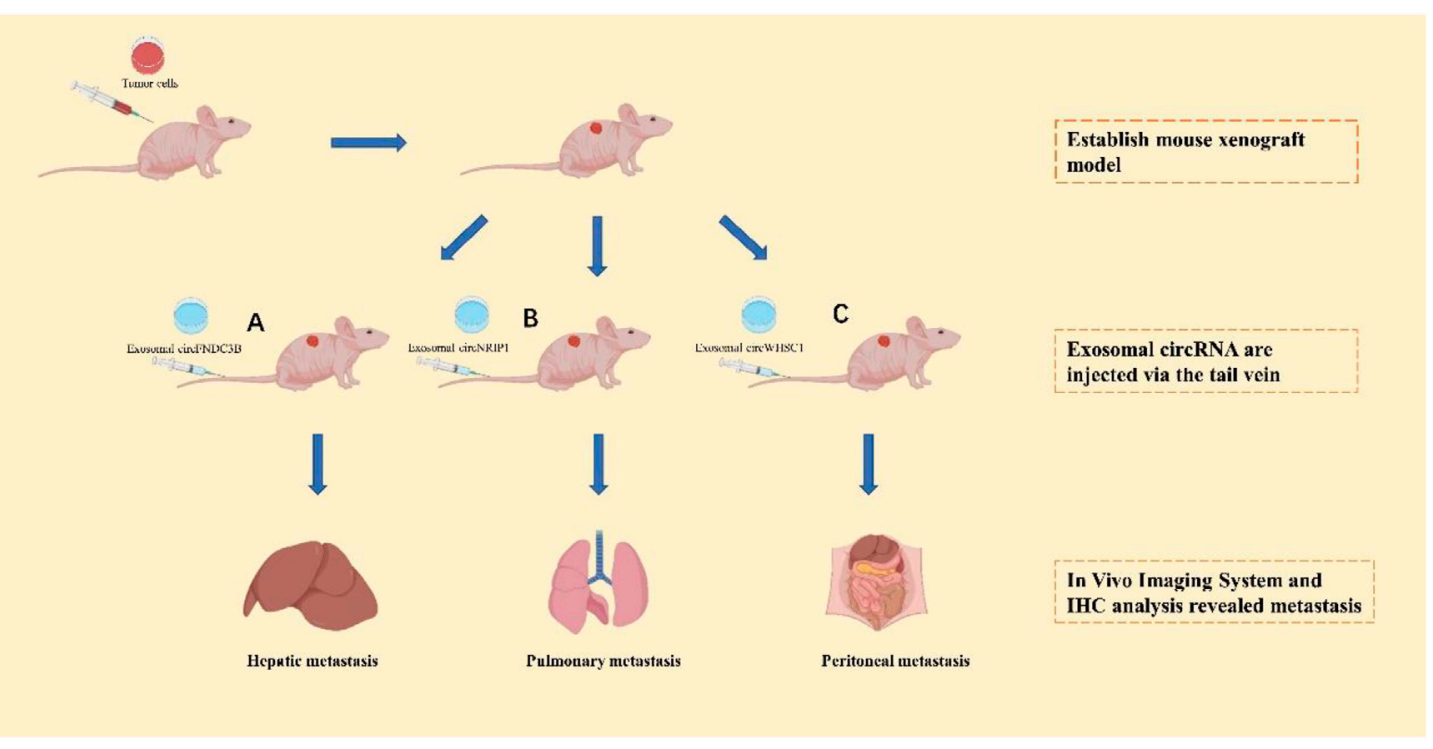

FIGURE 3 | The application of exosomal circRNAs as targets of tumors cell metastasis in vivo. After establishing the mouse xenograft model, exosomal circFNDC3B (A), exosomal circNRIP1 (B), and exosomal circWHSC1 (C) will be injected into the mouse xenograft model to analyze the organ-spectific metastasis.

exosomes. This result demonstrates that treatment with exosomal circFNDC3B inhibits CRC tumor growth, angiogenesis, and liver metastasis. Zhang et al. (Zhang et al., 2019) examined the role of exosomal circNRIP1 in distant metastasis through tail vein injection of GC cells co-cultured with circNRIP1-containing exosomes and normal control exosomes into $\mathrm{BALB} / \mathrm{c}$ nude mice. They found that lung and peritoneal metastases were more frequent in nude mice injected with circNRIP1containing exosomes than in mice treated with normal control exosomes, indicating that exosomal circNRIP1-treated GC cells exhibit greater metastatic potential. To investigate the role of exosomal circWHSC1 in the peritoneal dissemination of ovarian cancer, Zong et al. (Zong et al., 2019) injected CAOV3 cells intraperitoneally into nude mice to generate tumors, and then injected exosomes containing circWHSC1 or PBS every 2 days. They found that for the exosome-treated group, the number of peritoneal tumor nodules increased significantly and induced changes in their EMT. Recently, exosome-based transport systems have become an innovative platform for the transport of RNAs (siRNAs, microRNAs) or active-drug substances with enhanced specificity, and greater safety and stability compared to other carriers. Exosomes can be used as novel nanomaterials to deliver cargoes of circRNA inhibitors and agonists for suppressing tumor metastasis. However, we are still in the mapping stage of the clinical application of exosomal circRNAs, and no circRNA drug has entered clinical trials at the time of writing this review. MiRNAs are the most comprehensively understood non-coding RNAs, and several clinical studies have been conducted on miRNA-based interventions with respect to the progression of malignant tumors (Hong et al., 2020). A few examples are the first phase I clinical trial of cobomarsen (anti-miR-155 oligonucleotide) in patients with cutaneous T-cell lymphoma (CTCL) (Seto et al.,
2018) and a phase I clinical trial of a miR-16 analogue in patients with malignant pleural mesothelioma (van Zandwijk et al., 2017) or NSCLC. It is believed that, in the near future, circRNAs will also be used in clinical applications.

\section{FUTURE PERSPECTIVES}

With the rapid development of high-throughput sequencing technology, an increasing number of exosomal circRNAs have been discovered and identified, and have been studied and proven to play important roles in tumorigenesis. The covalently closed loop structure of circRNAs prevents them from being easily degraded by nucleases. Compared with exosomal proteins and ncRNAs (miRNA, lncRNA), exosomal circRNAs from tissues and blood are more conserved, stable, and exhibit greater target specificity, which is characteristic of tumor metastasis-related markers. In addition, as exosomal circRNAs play important regulatory roles in tumor metastasis, they have significant potential as important candidate targets for tumor metastasisrelated therapies, which opens new avenues for curing tumorigenic diseases. As the study of exosomal circRNAs is still in its infancy, many aspects remain limited. 1) The technology for the isolation and purification of exosomes is not yet sufficiently developed. Currently, there are four main methods for isolating and purifying extracellular vesicles, i.e., ultra-high speed centrifugation, ultrafiltration, precipitation, and immune-enrichment (van Niel et al., 2018). It is difficult to distinguish between exosomal and non-vesicular components using established purification methods, and this may affect subsequent experimental procedures involving exosomal circRNA, both in vivo and in vitro; 2) Although high-throughput sequencing techniques have identified many exosomal circRNAs 
that are aberrantly expressed in tumor tissues, their specific mechanisms of action and biological functions are still not fully understood; 3) The factors that determine the endogenous and exogenous nature of exosomes remain undetermined, which in part leads to the difficulty in using exosomal circRNAs as clinical markers of tumor metastasis; 4) Although an increasing number of studies have focused on exploring the use of exosomal circRNAs as biological markers for the diagnosis of certain tumors, only a few clinical trials have confirmed their feasibility.

In conclusion, this review has discussed the regulation of the occurrence and transport of exosomal circRNAs in various biological, physiological, or pathological processes by describing them as stellar molecules that have attracted much attention over recent years. Exosomal circRNAs are enriched in tumors and regulate tumor metastasis through mechanisms such as by acting as sponges for miRNAs, binding to proteins, and interacting with the TME. Although our current understanding of the functions of exosomal circRNAs is undoubtedly only the tip of the iceberg, the development of new technologies and assays would enable us to understand the regulatory mechanisms involving exosomal circRNAs, which will provide superior evidence for their use as early, novel markers of tumor metastasis, and as promising candidate therapeutic targets.

\section{REFERENCES}

Abdelmohsen, K., Panda, A. C., Munk, R., Grammatikakis, I., Dudekula, D. B., De, S., et al. (2017). Identification of HuR Target Circular RNAs Uncovers Suppression of PABPN1 Translation by CircPABPN1. RNA Biol. 14, 361-369. doi:10.1080/15476286.2017.1279788

Acharyya, S., and Massague, J. (2016). Arresting Supporters: Targeting Neutrophils in Metastasis. Cell Res 26, 273-274. doi:10.1038/cr.2016.17

Alenquer, M., and Amorim, M. (2015). Exosome Biogenesis, Regulation, and Function in Viral Infection. Viruses 7, 5066-5083. doi:10.3390/v7092862

Asghar, S., Litherland, G. J., Lockhart, J. C., Goodyear, C. S., and Crilly, A. (2020). Exosomes in Intercellular Communication and Implications for Osteoarthritis. Rheumatology (Oxford) 59, 57-68. doi:10.1093/rheumatology/kez462

Bai, H., Lei, K., Huang, F., Jiang, Z., and Zhou, X. (2019). Exo-circRNAs: a New Paradigm for Anticancer Therapy. Mol. Cancer 18, 56. doi:10.1186/s12943-0190986-2

Bakir, B., Chiarella, A. M., Pitarresi, J. R., and Rustgi, A. K. (2020). EMT, MET, Plasticity, and Tumor Metastasis. Trends Cel Biol. 30, 764-776. doi:10.1016/ j.tcb.2020.07.003

Bao, C., Lyu, D., and Huang, S. (2016). Circular RNA Expands its Territory. Mol. Cell Oncol. 3, e1084443. doi:10.1080/23723556.2015.1084443

Bebelman, M. P., Smit, M. J., Pegtel, D. M., and Baglio, S. R. (2018). Biogenesis and Function of Extracellular Vesicles in Cancer. Pharmacol. Ther. 188, 1-11. doi:10.1016/j.pharmthera.2018.02.013

Biswas, S. K., Sica, A., and Lewis, C. E. (2008). Plasticity of Macrophage Function during Tumor Progression: Regulation by Distinct Molecular Mechanisms. J. Immunol. 180, 2011-2017. doi:10.4049/jimmunol.180.4.2011

Braicu, C., Tomuleasa, C., Monroig, P., Cucuianu, A., Berindan-Neagoe, I., and Calin, G. A. (2015). Exosomes as Divine Messengers: Are They the Hermes of Modern Molecular Oncology? Cell Death Differ 22, 34-45. doi:10.1038/ cdd.2014.130

Chambers, A. F., Groom, A. C., and MacDonald, I. C. (2002). Dissemination and Growth of Cancer Cells in Metastatic Sites. Nat. Rev. Cancer 2, 563-572. doi:10.1038/nrc865

Chen, B., Sang, Y., Song, X., Zhang, D., Wang, L., Zhao, W., et al. (2021). Exosomal miR-500a-5p Derived from Cancer-Associated Fibroblasts Promotes Breast

\section{AUTHOR CONTRIBUTIONS}

PC and CO contributed to the conception and design of the study. $\mathrm{HZ}$ performed resource analysis, and wrote the first draft of the manuscript. All authors contributed to manuscript revision and read and approved the submitted version.

\section{FUNDING}

This study was supported by the National Natural Science Foundation of China (81903032), the China Postdoctoral Science Foundation (2020M672520), the Natural Science Foundation of Hunan Province (2019JJ40487, 2019JJ40497, and 2021JJ41013), the Research Program of Hunan Health Commission, China (202103030659) and the Youth Fund of Xiangya Hospital (2018Q011).

\section{SUPPLEMENTARY MATERIAL}

The Supplementary Material for this article can be found online at: https://www.frontiersin.org/articles/10.3389/fcell.2021.786224/ full\#supplementary-material

Cancer Cell Proliferation and Metastasis through Targeting USP28. Theranostics 11, 3932-3947. doi:10.7150/thno.53412

Chen, C., Luo, Y., He, W., Zhao, Y., Kong, Y., Liu, H., et al. (2019a). Exosomal Long Noncoding RNA LNMAT2 Promotes Lymphatic Metastasis in Bladder Cancer. J. Clin. Invest. 130, 404-421. doi:10.1172/JCI130892

Chen, W., Wang, H., Zhu, Z., Feng, J., and Chen, L. (2020b). Exosome-Shuttled circSHOC2 from IPASs Regulates Neuronal Autophagy and Ameliorates Ischemic Brain Injury via the miR-7670-3p/SIRT1 Axis. Mol. Ther. - Nucleic Acids 22, 657-672. doi:10.1016/j.omtn.2020.09.027

Chen, X., Chen, R.-X., Wei, W.-S., Li, Y.-H., Feng, Z.-H., Tan, L., et al. (2018). PRMT5 Circular RNA Promotes Metastasis of Urothelial Carcinoma of the Bladder through Sponging miR-30c to Induce Epithelial-Mesenchymal Transition. Clin. Cancer Res. 24, 6319-6330. doi:10.1158/1078-0432.CCR$18-1270$

Cocquerelle, C., Mascrez, B., Hétuin, D., and Bailleul, B. (1993). Mis-splicing Yields Circular RNA Molecules. FASEB J. 7, 155-160. doi:10.1096/fasebj.7.1.7678559

Dai, X., Chen, C., Yang, Q., Xue, J., Chen, X., Sun, B., et al. (2018). Exosomal circRNA_100284 from Arsenite-Transformed Cells, via microRNA-217 Regulation of EZH2, Is Involved in the Malignant Transformation of Human Hepatic Cells by Accelerating the Cell Cycle and Promoting Cell Proliferation. Cell Death Dis 9, 454. doi:10.1038/s41419-018-0485-1

Delpech, J.-C., Herron, S., Botros, M. B., and Ikezu, T. (2019). Neuroimmune Crosstalk through Extracellular Vesicles in Health and Disease. Trends Neurosciences 42, 361-372. doi:10.1016/j.tins.2019.02.007

Dignat-George, F., and Boulanger, C. M. (2011). The many Faces of Endothelial Microparticles. Atvb 31, 27-33. doi:10.1161/ATVBAHA.110.218123

Dou, Y., Cha, D. J., Franklin, J. L., Higginbotham, J. N., Jeppesen, D. K., Weaver, A. M., et al. (2016). Circular RNAs Are Down-Regulated in KRAS Mutant colon Cancer Cells and Can Be Transferred to Exosomes. Sci. Rep. 6, 37982. doi:10.1038/srep37982

Du, W. W., Zhang, C., Yang, W., Yong, T., Awan, F. M., and Yang, B. B. (2017). Identifying and Characterizing circRNA-Protein Interaction. Theranostics 7, 4183-4191. doi:10.7150/thno.21299

Fan, J., Xu, G., Chang, Z., Zhu, L., and Yao, J. (2020). miR-210 Transferred by Lung Cancer Cell-Derived Exosomes May Act as Proangiogenic Factor in CancerAssociated Fibroblasts by Modulating JAK2/STAT3 Pathway. Clin. Sci. (Lond) 134, 807-825. doi:10.1042/CS20200039 
Feng, W., Gong, H., Wang, Y., Zhu, G., Xue, T., Wang, Y., et al. (2019). circIFT80 Functions as a ceRNA of miR-1236-3p to Promote Colorectal Cancer Progression. Mol. Ther. - Nucleic Acids 18, 375-387. doi:10.1016/ j.omtn.2019.08.024

Fidler, I. J. (2003). The Pathogenesis of Cancer Metastasis: the 'seed and Soil' Hypothesis Revisited. Nat. Rev. Cancer 3, 453-458. doi:10.1038/nrc1098

Filipazzi, P., Bürdek, M., Villa, A., Rivoltini, L., and Huber, V. (2012). Recent Advances on the Role of Tumor Exosomes in Immunosuppression and Disease Progression. Semin. Cancer Biol. 22, 342-349. doi:10.1016/ j.semcancer.2012.02.005

Friedl, P., and Wolf, K. (2003). Tumour-cell Invasion and Migration: Diversity and Escape Mechanisms. Nat. Rev. Cancer 3, 362-374. doi:10.1038/nrc1075

Gao, L., Wang, L., Dai, T., Jin, K., Zhang, Z., Wang, S., et al. (2018). Tumor-derived Exosomes Antagonize Innate Antiviral Immunity. Nat. Immunol. 19, 233-245. doi:10.1038/s41590-017-0043-5

Gonda, A., Kabagwira, J., Senthil, G. N., and Wall, N. R. (2019). Internalization of Exosomes through Receptor-Mediated Endocytosis. Mol. Cancer Res. 17, 337-347. doi:10.1158/1541-7786.MCR-18-0891

Gu, C., Lu, H., and Qian, Z. (2020). Matrine Reduces the Secretion of Exosomal circSLC7A6 from Cancer-Associated Fibroblast to Inhibit Tumorigenesis of Colorectal Cancer by Regulating CXCR5. Biochem. Biophysical Res. Commun. 527, 638-645. doi:10.1016/j.bbrc.2020.04.142

Guo, C., Liu, J., Zhou, Q., Song, J., Zhang, Z., Li, Z., et al. (2020). Exosomal Noncoding RNAs and Tumor Drug Resistance. Cancer Res. 80, 4307-4313. doi:10.1158/0008-5472.CAN-20-0032

Gupta, G. P., and Massagué, J. (2006). Cancer Metastasis: Building a Framework. Cell 127, 679-695. doi:10.1016/j.cell.2006.11.001

Han, Y., Liu, Y., Zhang, B., and Yin, G. (2021). Exosomal circRNA 0001445 Promotes Glioma Progression through miRNA-127-5p/SNX5 Pathway. Aging 13, 13287-13299. doi:10.18632/aging.203013

Hanahan, D., and Coussens, L. M. (2012). Accessories to the Crime: Functions of Cells Recruited to the Tumor Microenvironment. Cancer Cell 21, 309-322. doi:10.1016/j.ccr.2012.02.022

Hanahan, D., and Weinberg, R. A. (2011). Hallmarks of Cancer: the Next Generation. Cell 144, 646-674. doi:10.1016/j.cell.2011.02.013

Hansen, T. B., Jensen, T. I., Clausen, B. H., Bramsen, J. B., Finsen, B., Damgaard, C. K., et al. (2013). Natural RNA Circles Function as Efficient microRNA Sponges. Nature 495, 384-388. doi:10.1038/nature11993

Hanson, P. I., and Cashikar, A. (2012). Multivesicular Body Morphogenesis. Annu. Rev. Cel Dev. Biol. 28, 337-362. doi:10.1146/annurev-cellbio-092910-154152

He, F., Zhong, X., Lin, Z., Lin, J., Qiu, M., Li, X., et al. (2020). Plasma Exohsa_circRNA_0056616: A Potential Biomarker for Lymph Node Metastasis in Lung Adenocarcinoma. J. Cancer 11, 4037-4046. doi: $10.7150 /$ jca. 30360

He, L., Zhu, W., Chen, Q., Yuan, Y., Wang, Y., Wang, J., et al. (2019). Ovarian Cancer Cell-Secreted Exosomal miR-205 Promotes Metastasis by Inducing Angiogenesis. Theranostics 9, 8206-8220. doi:10.7150/thno.37455

He, X., Kuang, G., Wu, Y., and Ou, C. (2021a). Emerging Roles of Exosomal miRNAs in Diabetes Mellitus. Clin. Translational Med. 11, e468. doi:10.1002/ ctm2.468

He, X., Yu, B., Kuang, G., Wu, Y., Zhang, M., Cao, P., et al. (2021b). Long Noncoding RNA DLEU2 Affects the Proliferative and Invasive Ability of Colorectal Cancer Cells. J. Cancer 12, 428-437. doi:10.7150/jca.48423

Hentze, M. W., and Preiss, T. (2013). Circular RNAs: Splicing's enigma Variations. EMBO J. 32, 923-925. doi:10.1038/emboj.2013.53

Hessvik, N. P., and Llorente, A. (2018). Current Knowledge on Exosome Biogenesis and Release. Cell. Mol. Life Sci. 75, 193-208. doi:10.1007/s00018-017-2595-9

Hinshaw, D. C., and Shevde, L. A. (2019). The Tumor Microenvironment Innately Modulates Cancer Progression. Cancer Res. 79, 4557-4566. doi:10.1158/00085472.CAN-18-3962

Holmgren, L., O'Reilly, M. S., and Folkman, J. (1995). Dormancy of Micrometastases: Balanced Proliferation and Apoptosis in the Presence of Angiogenesis Suppression. Nat. Med. 1, 149-153. doi:10.1038/ nm0295-149

Hong, D. S., Kang, Y.-K., Borad, M., Sachdev, J., Ejadi, S., Lim, H. Y., et al. (2020). Phase 1 Study of MRX34, a Liposomal miR-34a Mimic, in Patients with Advanced Solid Tumours. Br. J. Cancer 122, 1630-1637. doi:10.1038/ s41416-020-0802-1
Hou, J., Jiang, W., Zhu, L., Zhong, S., Zhang, H., Li, J., et al. (2018). Circular RNAs and Exosomes in Cancer: a Mysterious Connection. Clin. Transl Oncol. 20, 1109-1116. doi:10.1007/s12094-018-1839-y

Hu, J. L., Wang, W., Lan, X. L., Zeng, Z. C., Liang, Y. S., Yan, Y. R., et al. (2019a). CAFs Secreted Exosomes Promote Metastasis and Chemotherapy Resistance by Enhancing Cell Stemness and Epithelial-Mesenchymal Transition in Colorectal Cancer. Mol. Cancer 18, 91. doi:10.1186/s12943-019-1019-x

Hu, K., Liu, X., Li, Y., Li, Q., Xu, Y., Zeng, W., et al. (2020). Exosomes Mediated Transfer of Circ_UBE2D2 Enhances the Resistance of Breast Cancer to Tamoxifen by Binding to MiR-200a-3p. Med. Sci. Monit. 26. doi:10.12659/ MSM.922253

Hu, Y., Zhao, Y., Shi, C., Ren, P., Wei, B., Guo, Y., et al. (2019b). A Circular RNA from APC Inhibits the Proliferation of Diffuse Large B-Cell Lymphoma by Inactivating $\mathrm{Wnt} / \beta$-Catenin Signaling via Interacting with TET 1 and miR-888. Aging 11, 8068-8084. doi:10.18632/aging.102122

Huang, A., Zheng, H., Wu, Z., Chen, M., and Huang, Y. (2020a). Circular RNAProtein Interactions: Functions, Mechanisms, and Identification. Theranostics 10, 3503-3517. doi:10.7150/thno.42174

Huang, X.-Y., Huang, Z.-L., Huang, J., Xu, B., Huang, X.-Y., Xu, Y.-H., et al. (2020c). Exosomal circRNA-100338 Promotes Hepatocellular Carcinoma Metastasis via Enhancing Invasiveness and Angiogenesis. J. Exp. Clin. Cancer Res. 39, 20. doi:10.1186/s13046-020-1529-9

Huang, X., Wu, B., Chen, M., Hong, L., Kong, P., Wei, Z., et al. (2020b). Depletion of Exosomal circLDLR in Follicle Fluid Derepresses miR-1294 Function and Inhibits Estradiol Production via CYP19A1 in Polycystic Ovary Syndrome. Aging 12, 15414-15435. doi:10.18632/aging.103602

Jeck, W. R., Sorrentino, J. A., Wang, K., Slevin, M. K., Burd, C. E., Liu, J., et al. (2013). Circular RNAs Are Abundant, Conserved, and Associated with ALU Repeats. RNA 19, 141-157. doi:10.1261/rna.035667.112

Jiang, M.-C., Ni, J.-J., Cui, W.-Y., Wang, B.-Y., and Zhuo, W. (2019). Emerging Roles of lncRNA in Cancer and Therapeutic Opportunities. Am. J. Cancer Res. 9 (7), 1354-1366.

Jin, M., Shi, C., Yang, C., Liu, J., and Huang, G. (2019). Upregulated circRNA ARHGAP10 Predicts an Unfavorable Prognosis in NSCLC through Regulation of the miR-150-5p/GLUT-1 Axis. Mol. Ther. - Nucleic Acids 18, 219-231. doi:10.1016/j.omtn.2019.08.016

Joyce, J. A., and Pollard, J. W. (2009). Microenvironmental Regulation of Metastasis. Nat. Rev. Cancer 9, 239-252. doi:10.1038/nrc2618

Kalluri, R. (2016). The Biology and Function of Exosomes in Cancer. J. Clin. Invest. 126, 1208-1215. doi:10.1172/JCI81135

Kalra, H., Drummen, G., and Mathivanan, S. (2016). Focus on Extracellular Vesicles: Introducing the Next Small Big Thing. Ijms 17, 170. doi:10.3390/ ijms 17020170

Kato, T., Noma, K., Ohara, T., Kashima, H., Katsura, Y., Sato, H., et al. (2018). Cancer-Associated Fibroblasts Affect Intratumoral CD8+ and FoxP3+ T Cells via IL6 in the Tumor Microenvironment. Clin. Cancer Res. 24, 4820-4833. doi:10.1158/1078-0432.CCR-18-0205

Katopodi, T., Petanidis, S., Domvri, K., Zarogoulidis, P., Anestakis, D., Charalampidis, C., et al. (2021). Kras-driven Intratumoral Heterogeneity Triggers Infiltration of M2 Polarized Macrophages via the circHIPK3/PTK2 Immunosuppressive Circuit. Sci. Rep. 11, 15455. doi:10.1038/s41598-02194671-x

Kim, S. H., Bianco, N. R., Shufesky, W. J., Morelli, A. E., and Robbins, P. D. (2007), MHC Class II+ Exosomes in Plasma Suppress Inflammation in an Antigenspecific and Fas ligand/Fas-dependent Manner. J. Immunol. 179, 2235-2241. doi:10.4049/jimmunol.179.4.2235

Kolakofsky, D. (1976). Isolation and Characterization of Sendai Virus DI-RNAs. Cell 8, 547-555. doi:10.1016/0092-8674(76)90223-3

Kowal, J., Arras, G., Colombo, M., Jouve, M., Morath, J. P., Primdal-Bengtson, B., et al. (2016). Proteomic Comparison Defines Novel Markers to Characterize Heterogeneous Populations of Extracellular Vesicle Subtypes. Proc. Natl. Acad. Sci. USA 113, E968-E977. doi:10.1073/pnas.1521230113

Kugeratski, F. G., and Kalluri, R. (2021). Exosomes as Mediators of Immune Regulation and Immunotherapy in Cancer. FEBS J. 288, 10-35. doi:10.1111/ febs. 15558

Langley, R. R., and Fidler, I. J. (2011). The Seed and Soil Hypothesis Revisited-The Role of Tumor-Stroma Interactions in Metastasis to Different Organs. Int. J. Cancer 128, 2527-2535. doi:10.1002/ijc.26031 
Lasda, E., and Parker, R. (2016). Circular RNAs Co-precipitate with Extracellular Vesicles: A Possible Mechanism for circRNA Clearance. PLoS One 11, e0148407. doi:10.1371/journal.pone.0148407

Lasda, E., and Parker, R. (2014). Circular RNAs: Diversity of Form and Function. RNA 20, 1829-1842. doi:10.1261/rna.047126.114

Lazebnik, Y. (2010). What Are the Hallmarks of Cancer? Nat. Rev. Cancer 10, 232-233. doi:10.1038/nrc2827

Legnini, I., Di Timoteo, G., Rossi, F., Morlando, M., Briganti, F., Sthandier, O., et al. (2017). Circ-ZNF609 Is a Circular RNA that Can Be Translated and Functions in Myogenesis. Mol. Cel 66, 22-37. doi:10.1016/j.molcel.2017.02.017

Lei, M., Zheng, G., Ning, Q., Zheng, J., and Dong, D. (2020). Translation and Functional Roles of Circular RNAs in Human Cancer. Mol. Cancer 19, 30. doi:10.1186/s12943-020-1135-7

Li, B. L., DuLu, G. Q., and Wan, X. P. (2019). Loss of Exosomal miR-148b from Cancer-associated Fibroblasts Promotes Endometrial Cancer Cell Invasion and Cancer Metastasis. J. Cel Physiol 234, 2943-2953. doi:10.1002/jcp.27111

Li, J., Li, Z., Jiang, P., Peng, M., Zhang, X., Chen, K., et al. (2018). Circular RNA IARS (Circ-IARS) Secreted by Pancreatic Cancer Cells and Located within Exosomes Regulates Endothelial Monolayer Permeability to Promote Tumor Metastasis. J. Exp. Clin. Cancer Res. 37, 177. doi:10.1186/s13046-018-0822-3

Li, L., Li, W., Chen, N., Zhao, H., Xu, G., Zhao, Y., et al. (2019a). FLI1 Exonic Circular RNAs as a Novel Oncogenic Driver to Promote Tumor Metastasis in Small Cell Lung Cancer. Clin. Cancer Res. 25, 1302-1317. doi:10.1158/1078-0432.CCR-18-1447

Li, R., Wang, Y., Zhang, X., Feng, M., Ma, J., Li, J., et al. (2019b). Exosome-mediated Secretion of LOXL4 Promotes Hepatocellular Carcinoma Cell Invasion and Metastasis. Mol. Cancer 18, 18. doi:10.1186/s12943-019-0948-8

Li, T., Sun, X., and Chen, L. (2020a). Exosome Circ_0044516 Promotes Prostate Cancer Cell Proliferation and Metastasis as a Potential Biomarker. J. Cel Biochem 121, 2118-2126. doi:10.1002/jcb.28239

Li, Y.-Y., Tao, Y.-W., Gao, S., Li, P., Zheng, J.-M., Zhang, S.-E., et al. (2018a). Cancer-associated Fibroblasts Contribute to Oral Cancer Cells Proliferation and Metastasis via Exosome-Mediated Paracrine miR-34a-5p. EBioMedicine 36, 209-220. doi:10.1016/j.ebiom.2018.09.006

Li, Y., Zang, H., Zhang, X., and Huang, G. (2020b). Exosomal Circ-Znf652 Promotes Cell Proliferation, Migration, Invasion and Glycolysis in Hepatocellular Carcinoma via miR-29a-3p/GUCD1 Axis. CMAR 12, 7739-7751. doi:10.2147/CMAR.S259424

Li, Y., Zheng, Q., Bao, C., Li, S., Guo, W., Zhao, J., et al. (2015). Circular RNA Is Enriched and Stable in Exosomes: a Promising Biomarker for Cancer Diagnosis. Cel Res 25, 981-984. doi:10.1038/cr.2015.82

Li, Z., Yanfang, W., Li, J., Jiang, P., Peng, T., Chen, K., et al. (2018b). Tumorreleased Exosomal Circular RNA PDE8A Promotes Invasive Growth via the miR-338/MACC1/MET Pathway in Pancreatic Cancer. Cancer Lett. 432, 237-250. doi:10.1016/j.canlet.2018.04.035

Liang, J., Wu, X., Sun, S., Chen, P., Liang, X., Wang, J., et al. (2018). Circular RNA Expression Profile Analysis of Severe Acne by RNA -Seq and Bioinformatics. J. Eur. Acad. Dermatol. Venereol. 32, 1986-1992. doi:10.1111/jdv.14948

Liang, Z.-Z., Guo, C., Zou, M.-M., Meng, P., and Zhang, T.-T. (2020). circRNAmiRNA-mRNA Regulatory Network in Human Lung Cancer: an Update. Cancer Cel Int 20, 173. doi:10.1186/s12935-020-01245-4

Liao, Z., Nie, H., Wang, Y., Luo, J., Zhou, J., and Ou, C. (2021). The Emerging Landscape of Long Non-coding RNAs in Colorectal Cancer Metastasis. Front. Oncol. 11, 641343. doi:10.3389/fonc.2021.641343

Linli, T., Jing, C., Hui, J., Jiarui, Z., Xiuxia, R., Xinyu, L., et al. (2019). CircRASSF2 Promotes Laryngeal Squamous Cell Carcinoma Progression by Regulating the miR-302b-3p/IGF-1R axis. Clin. Sci. 133, 1053-1066. doi:10.1042/CS20190110

Liu, C., Ge, H.-M., Liu, B.-H., Dong, R., Shan, K., Chen, X., et al. (2019). Targeting Pericyte-Endothelial Cell Crosstalk by Circular RNA-cPWWP2A Inhibition Aggravates Diabetes-Induced Microvascular Dysfunction. Proc. Natl. Acad. Sci. USA 116, 7455-7464. doi:10.1073/pnas.1814874116

Liu, D., Kang, H., Gao, M., Jin, L., Zhang, F., Chen, D., et al. (2020). Exosometransmitted circ_MMP2 Promotes Hepatocellular Carcinoma Metastasis by Upregulating MMP2. Mol. Oncol. 14, 1365-1380. doi:10.1002/1878-0261.12637

Liu, S., Lin, Z., Rao, W., Zheng, J., Xie, Q., Lin, Y., et al. (2021). Upregulated Expression of Serum Exosomal Hsa_circ_0026611 Is Associated with Lymph Node Metastasis and Poor Prognosis of Esophageal Squamous Cell Carcinoma. J. Cancer 12, 918-926. doi:10.7150/jca.50548
Liu, Y., and Cao, X. (2016). Characteristics and Significance of the Pre-metastatic Niche. Cancer Cell 30, 668-681. doi:10.1016/j.ccell.2016.09.011

Lu, Q., Wang, X., Zhu, J., Fei, X., Chen, H., and Li, C. (2020). Hypoxic TumorDerived Exosomal Circ0048117 Facilitates M2 Macrophage Polarization Acting as miR-140 Sponge in Esophageal Squamous Cell Carcinoma. OTT Vol. 13, 11883-11897. doi:10.2147/OTT.S284192

Luo, Y., Liu, F., Guo, J., and Gui, R. (2020a). Upregulation of Circ_0000199 in Circulating Exosomes Is Associated with Survival Outcome in OSCC. Sci. Rep. 10, 13739. doi:10.1038/s41598-020-70747-y

Luo, Y., Ma, J., Liu, F., Guo, J., and Gui, R. (2020b). Diagnostic Value of Exosomal circMYC in Radioresistant Nasopharyngeal Carcinoma. Head \& Neck 42, 3702-3711. doi:10.1002/hed.26441

Luo, Z., Wang, Q., Lau, W. B., Lau, B., Xu, L., Zhao, L., et al. (2016). Tumor Microenvironment: The Culprit for Ovarian Cancer Metastasis? Cancer Lett. 377, 174-182. doi:10.1016/j.canlet.2016.04.038

Ma, R., Ye, X., Cheng, H., Cui, H., and Chang, X. (2021). Tumor-derived Exosomal circRNA051239 Promotes Proliferation and Migration of Epithelial Ovarian Cancer. Am. J. Transl Res. 13, 1125-1139.

Maishi, N., and Hida, K. (2017). Tumor Endothelial Cells Accelerate Tumor Metastasis. Cancer Sci. 108, 1921-1926. doi:10.1111/cas.13336

Maniecki, M. B., Etzerodt, A., Ulhøi, B. P., Steiniche, T., Borre, M., Dyrskjøt, L., et al. (2012). Tumor-promoting Macrophages Induce the Expression of the Macrophage-specific Receptor CD163 in Malignant Cells. Int. J. Cancer 131, 2320-2331. doi:10.1002/ijc.27506

Mantovani, A., and Sica, A. (2010). Macrophages, Innate Immunity and Cancer: Balance, Tolerance, and Diversity. Curr. Opin. Immunol. 22, 231-237. doi:10.1016/j.coi.2010.01.009

Marx, V. (2013). Tracking Metastasis and Tricking Cancer. Nature 494, 133-138. doi:10.1038/494131a

Massagué, J., and Obenauf, A. C. (2016). Metastatic Colonization by Circulating Tumour Cells. Nature 529, 298-306. doi:10.1038/nature17038

Mathivanan, S., Ji, H., and Simpson, R. J. (2010). Exosomes: Extracellular Organelles Important in Intercellular Communication. J. Proteomics 73, 1907-1920. doi:10.1016/j.jprot.2010.06.006

Mattiuzzi, C., and Lippi, G. (2020). Cancer Statistics: a Comparison between World Health Organization (WHO) and Global Burden of Disease (GBD). Eur. J. Public Health 30, 1026-1027. doi:10.1093/eurpub/ckz216

McAllister, S. S., and Weinberg, R. A. (2014). The Tumour-Induced Systemic Environment as a Critical Regulator of Cancer Progression and Metastasis. Nat. Cel Biol 16, 717-727. doi:10.1038/ncb3015

McGuirk, P., and Mills, K. H. G. (2002). Pathogen-specific Regulatory T Cells Provoke a Shift in the Th1/Th2 Paradigm in Immunity to Infectious Diseases. Trends Immunol. 23, 450-455. doi:10.1016/s1471-4906(02) 02288-3

Meldolesi, J. (2018). Exosomes and Ectosomes in Intercellular Communication. Curr. Biol. 28, R435-R444. doi:10.1016/j.cub.2018.01.059

Memczak, S., Jens, M., Elefsinioti, A., Torti, F., Krueger, J., Rybak, A., et al. (2013). Circular RNAs Are a Large Class of Animal RNAs with Regulatory Potency. Nature 495, 333-338. doi:10.1038/nature11928

Meng, W., Hao, Y., He, C., Li, L., and Zhu, G. (2019). Exosome-orchestrated Hypoxic Tumor Microenvironment. Mol. Cancer 18, 57. doi:10.1186/s12943019-0982-6

Nguyen, D. X., Bos, P. D., and Massagué, J. (2009). Metastasis: from Dissemination to Organ-specific Colonization. Nat. Rev. Cancer 9, 274-284. doi:10.1038/ $\operatorname{nrc} 2622$

Nie, H., Wang, Y., Liao, Z., Zhou, J., and Ou, C. (2020). The Function and Mechanism of Circular RNAs in Gastrointestinal Tumours. Cell Prolif 53, e12815. doi:10.1111/cpr.12815

Nishiyama, M., Tsunedomi, R., Yoshimura, K., Hashimoto, N., Matsukuma, S., Ogihara, H., et al. (2018). Metastatic Ability and the Epithelial-mesenchymal Transition in Induced Cancer Stem-like Hepatoma Cells. Cancer Sci. 109, 1101-1109. doi:10.1111/cas.13527

Ou, C., Sun, Z., He, X., Li, X., Fan, S., Zheng, X., et al. (2020). Targeting YAP1/ LINC00152/FSCN1 Signaling Axis Prevents the Progression of Colorectal Cancer. Adv. Sci. 7, 1901380. doi:10.1002/advs.201901380

Paget, S. (1989). The Distribution of Secondary Growths in Cancer of the Breast. 1889. Cancer Metastasis Rev. 8, 98-101. 
Pan, B., Qin, J., Liu, X., He, B., Wang, X., Pan, Y., et al. (2019). Identification of Serum Exosomal Hsa-Circ-0004771 as a Novel Diagnostic Biomarker of Colorectal Cancer. Front. Genet. 10, 1096. doi:10.3389/fgene.2019.01096

Perez de Acha, O., Rossi, M., and Gorospe, M. (2020). Circular RNAs in Blood Malignancies. Front. Mol. Biosci. 7, 109. doi:10.3389/fmolb.2020.00109

Psaila, B., and Lyden, D. (2009). The Metastatic Niche: Adapting the Foreign Soil. Nat. Rev. Cancer 9, 285-293. doi:10.1038/nrc2621

Qian, B.-Z., and Pollard, J. W. (2010). Macrophage Diversity Enhances Tumor Progression and Metastasis. Cell 141, 39-51. doi:10.1016/j.cell.2010.03.014

Quail, D. F., and Joyce, J. A. (2013). Microenvironmental Regulation of Tumor Progression and Metastasis. Nat. Med. 19, 1423-1437. doi:10.1038/nm.3394

Ribelles, N., Santonja, A., Pajares, B., Llácer, C., and Alba, E. (2014). The Seed and Soil Hypothesis Revisited: Current State of Knowledge of Inherited Genes on Prognosis in Breast Cancer. Cancer Treat. Rev. 40, 293-299. doi:10.1016/ j.ctrv.2013.09.010

Ridge, S. M., Sullivan, F. J., and Glynn, S. A. (2017). Mesenchymal Stem Cells: Key Players in Cancer Progression. Mol. Cancer 16, 31. doi:10.1186/s12943-0170597-8

Salzman, J., Gawad, C., Wang, P. L., Lacayo, N., and Brown, P. O. (2012). Circular RNAs Are the Predominant Transcript Isoform from Hundreds of Human Genes in Diverse Cell Types. PLoS One 7, e30733. doi:10.1371/ journal.pone. 0030733

Scully, O. J., Bay, B. H., Yip, G., and Yu, Y. (2012). Breast Cancer Metastasis. Cancer Genomics Proteomics 9, 311-320.

Seto, A. G., Beatty, X., Lynch, J. M., Hermreck, M., Tetzlaff, M., Duvic, M., et al. (2018). Cobomarsen, an Oligonucleotide Inhibitor of miR-155, Co-ordinately Regulates Multiple Survival Pathways to Reduce Cellular Proliferation and Survival in Cutaneous T-Cell Lymphoma. Br. J. Haematol. 183, 428-444. doi:10.1111/bjh.15547

Shang, A., Gu, C., Wang, W., Wang, X., Sun, J., Zeng, B., et al. (2020). Exosomal circPACRGL Promotes Progression of Colorectal Cancer via the miR-142-3p/ miR-506-3p- TGF-B1 axis. Mol. Cancer 19, 117. doi:10.1186/s12943-02001235-0

Shang, B.-Q., Li, M.-L., Quan, H.-y., Hou, P.-F., Li, Z.-W., Chu, S.-F., et al. (2019). Functional Roles of Circular RNAs during Epithelial-To-Mesenchymal Transition. Mol. Cancer 18, 138. doi:10.1186/s12943-019-1071-6

Shi, H., Huang, S., Qin, M., Xue, X., Guo, X., Jiang, L., et al. (2021). Exosomal Circ_0088300 Derived from Cancer-Associated Fibroblasts Acts as a miR-1305 Sponge and Promotes Gastric Carcinoma Cell Tumorigenesis. Front. Cel Dev. Biol. 9, 676319. doi:10.3389/fcell.2021.676319

Shi, X., Wang, B., Feng, X., Xu, Y., Lu, K., and Sun, M. (2020). circRNAs and Exosomes: A Mysterious Frontier for Human Cancer. Mol. Ther. - Nucleic Acids 19, 384-392. doi:10.1016/j.omtn.2019.11.023

Song, J., Chen, Z.-H., Zheng, C.-J., Song, K.-H., Xu, G.-Y., Xu, S., et al. (2020). Exosome-Transported circRNA_0000253 Competitively Adsorbs MicroRNA141-5p and Increases IDD. Mol. Ther. - Nucleic Acids 21, 1087-1099. doi:10.1016/j.omtn.2020.07.039

Spaeth, E., Klopp, A., Dembinski, J., Andreeff, M., and Marini, F. (2008). Inflammation and Tumor Microenvironments: Defining the Migratory Itinerary of Mesenchymal Stem Cells. Gene Ther. 15, 730-738. doi:10.1038/ gt.2008.39

Stoletov, K., Beatty, P. H., and Lewis, J. D. (2020). Novel Therapeutic Targets for Cancer Metastasis. Expert Rev. Anticancer Ther. 20, 97-109. doi:10.1080/ 14737140.2020 .1718496

Sun, L., Chen, L., and Li, H. (2019a). Checkpoint-modulating Immunotherapies in Tumor Treatment: Targets, Drugs, and Mechanisms. Int. Immunopharmacology 67, 160-175. doi:10.1016/j.intimp.2018.12.006

Sun, Y.-M., Wang, W.-T., Zeng, Z.-C., Chen, T.-Q., Han, C., Pan, Q., et al. (2019b). circMYBL2, a circRNA from MYBL2, Regulates FLT3 Translation by Recruiting PTBP1 to Promote FLT3-ITD AML Progression. Blood 134, 1533-1546. doi:10.1182/blood.2019000802

Sun, Z., Wang, Q., Yu, X., Ou, C., Yao, L., Liu, K., et al. (2015). Risk Factors Associated with Splenic Hilar Lymph Node Metastasis in Patients with Advanced Gastric Cancer in Northwest China. Int. J. Clin. Exp. Med. 8, 21358-21364

Suzuki, M., Mose, E. S., Montel, V., and Tarin, D. (2006). Dormant Cancer Cells Retrieved from Metastasis-free Organs Regain Tumorigenic and Metastatic Potency. Am. J. Pathol. 169, 673-681. doi:10.2353/ajpath.2006.060053
Tao, S.-C., Huang, J.-Y., Gao, Y., Li, Z.-X., Wei, Z.-Y., Dawes, H., et al. (2021). Small Extracellular Vesicles in Combination with Sleep-Related circRNA3503: A Targeted Therapeutic Agent with Injectable Thermosensitive Hydrogel to Prevent Osteoarthritis. Bioactive Mater. 6, 4455-4469. doi:10.1016/ j.bioactmat.2021.04.031

Tarin, D. (2011). Cell and Tissue Interactions in Carcinogenesis and Metastasis and Their Clinical Significance. Semin. Cancer Biol. 21, 72-82. doi:10.1016/ j.semcancer.2010.12.006

Théry, C., Zitvogel, L., and Amigorena, S. (2002). Exosomes: Composition, Biogenesis and Function. Nat. Rev. Immunol. 2, 569-579. doi:10.1038/nri855

Tlsty, T. D., and Coussens, L. M. (2006). Tumor Stroma and Regulation of Cancer Development. Annu. Rev. Pathol. Mech. Dis. 1, 119-150. doi:10.1146/ annurev.pathol.1.110304.100224

Vakhshiteh, F., Atyabi, F., and Ostad, S. N. (2019). Mesenchymal Stem Cell Exosomes: a Two-Edged Sword in Cancer Therapy. IJN 14, 2847-2859. doi:10.2147/IJN.S200036

van Niel, G., D’Angelo, G., and Raposo, G. (2018). Shedding Light on the Cell Biology of Extracellular Vesicles. Nat. Rev. Mol. Cel Biol 19, 213-228. doi:10.1038/nrm.2017.125

van Zandwijk, N., Pavlakis, N., Kao, S. C., Linton, A., Boyer, M. J., Clarke, S., et al. (2017). Safety and Activity of microRNA-Loaded Minicells in Patients with Recurrent Malignant Pleural Mesothelioma: a First-In-Man, Phase 1, OpenLabel, Dose-Escalation Study. Lancet Oncol. 18, 1386-1396. doi:10.1016/S14702045(17)30621-6

Verduci, L., Strano, S., Yarden, Y., and Blandino, G. (2019). The Circ RNA -micro RNA Code: Emerging Implications for Cancer Diagnosis and Treatment. Mol. Oncol. 13, 669-680. doi:10.1002/1878-0261.12468

Wang, G., Liu, W., Zou, Y., Wang, G., Deng, Y., Luo, J., et al. (2019a). Three Isoforms of Exosomal circPTGR1 Promote Hepatocellular Carcinoma Metastasis via the miR449a-MET Pathway. EBioMedicine 40, 432-445. doi:10.1016/j.ebiom.2018.12.062

Wang, H., Wei, M., Kang, Y., Xing, J., and Zhao, Y. (2020a). Circular RNA circ_PVT1 Induces Epithelial-Mesenchymal Transition to Promote Metastasis of Cervical Cancer. aging 12, 20139-20151. doi:10.18632/aging.103679

Wang, J., Wu, X., Shen, P., Wang, J., Shen, Y., Shen, Y., et al. (2020b). Applications of Inorganic Nanomaterials in Photothermal Therapy Based on Combinational Cancer Treatment. Ijn 15, 1903-1914. doi:10.2147/IJN.S239751

Wang, S., Hu, Y., Lv, X., Li, B., Gu, D., Li, Y., et al. (2019b). Circ-0000284 Arouses Malignant Phenotype of Cholangiocarcinoma Cells and Regulates the Biological Functions of Peripheral Cells through Cellular Communication. Clin. Sci. (Lond) 133, 1935-1953. doi:10.1042/CS20190589

Wang, S., Zhan, J., Lin, X., Wang, Y., Wang, Y., and Liu, Y. (2020c). CircRNA -0077930 from Hyperglycaemia-stimulated Vascular Endothelial Cell Exosomes Regulates Senescence in Vascular Smooth Muscle Cells. Cell Biochem Funct 38, 1056-1068. doi:10.1002/cbf.3543

Wang, Y., Gao, R., Li, J., Tang, S., Li, S., Tong, Q., et al. (2021). Downregulation of Hsa_circ_0074854 Suppresses the Migration and Invasion in Hepatocellular Carcinoma via Interacting with $\mathrm{HuR}$ and via Suppressing Exosomes-Mediated Macrophage M2 Polarization. IJN 16, 2803-2818. doi:10.2147/IJN.S284560

Wang, Y., Nie, H., He, X., Liao, Z., Zhou, Y., Zhou, J., et al. (2020d). The Emerging Role of Super Enhancer-Derived Noncoding RNAs in Human Cancer. Theranostics 10, 11049-11062. doi:10.7150/thno.49168

Wang, Y., Zhao, R., Shen, C., Liu, W., Yuan, J., Li, C., et al. (2020e). Exosomal CircHIPK3 Released from Hypoxia-Induced Cardiomyocytes Regulates Cardiac Angiogenesis after Myocardial Infarction. Oxidative Med. Cell Longevity 2020, 1-19. doi:10.1155/2020/8418407

Whiteside, T. L. (2016). Tumor-Derived Exosomes and Their Role in Cancer Progression. Adv. Clin. Chem. 74, 103-141. doi:10.1016/bs.acc.2015.12.005

Wilusz, J. E., and Sharp, P. A. (2013). A Circuitous Route to Noncoding RNA. Science 340, 440-441. doi:10.1126/science.1238522

Wortzel, I., Dror, S., Kenific, C. M., and Lyden, D. (2019). Exosome-Mediated Metastasis: Communication from a Distance. Developmental Cel 49, 347-360. doi:10.1016/j.devcel.2019.04.011

Wu, Q., Zhou, L., Lv, D., Zhu, X., and Tang, H. (2019). Exosome-mediated Communication in the Tumor Microenvironment Contributes to Hepatocellular Carcinoma Development and Progression. J. Hematol. Oncol. 12, 53. doi:10.1186/s13045-019-0739-0 
Xiao, H., and Shi, J. (2020). Exosomal Circular RNA_400068 Promotes the Development of Renal Cell Carcinoma via the miR-210-5p/SOCS1 axis. Mol. Med. Rep. 22, 4810-4820. doi:10.3892/mmr.2020.11541

Xiao, Y., and Yu, D. (2021). Tumor Microenvironment as a Therapeutic Target in Cancer. Pharmacol. Ther. 221, 107753. doi:10.1016/j.pharmthera.2020.107753

Xie, M., Yu, T., Jing, X., Ma, L., Fan, Y., Yang, F., et al. (2020). Exosomal circSHKBP1 Promotes Gastric Cancer Progression via Regulating the miR582-3p/HUR/VEGF axis and Suppressing HSP90 Degradation. Mol. Cancer 19, 112. doi:10.1186/s12943-020-01208-3

Xu, L., Liao, W.-L., Lu, Q.-J., Zhang, P., Zhu, J., and Jiang, G.-N. (2021a). Hypoxic Tumor-Derived Exosomal Circular RNA SETDB1 Promotes Invasive Growth and EMT via the miR-7/Sp1 axis in Lung Adenocarcinoma. Mol. Ther. - Nucleic Acids 23, 1078-1092. doi:10.1016/j.omtn.2021.01.019

Xu, R., Rai, A., Chen, M., Suwakulsiri, W., Greening, D. W., and Simpson, R. J. (2018). Extracellular Vesicles in Cancer - Implications for Future Improvements in Cancer Care. Nat. Rev. Clin. Oncol. 15, 617-638. doi:10.1038/s41571-018-0036-9

Xu, Y., Leng, K., Yao, Y., Kang, P., Liao, G., Han, Y., et al. (2021b). A Circular RNA, Cholangiocarcinoma-Associated Circular RNA 1, Contributes to Cholangiocarcinoma Progression, Induces Angiogenesis, and Disrupts Vascular Endothelial Barriers. Hepatology 73, 1419-1435. doi:10.1002/hep.31493

Yang, B., Teng, F., Chang, L., Wang, J., Liu, D.-L., Cui, Y.-S., et al. (2021a). Tumorderived Exosomal circRNA_102481 Contributes to EGFR-TKIs Resistance via the miR-30a-5p/ROR1 axis in Non-small Cell Lung Cancer. Aging 13, 13264-13286. doi:10.18632/aging.203011

Yang, H., Zhang, H., Yang, Y., Wang, X., Deng, T., Liu, R., et al. (2020). Hypoxia Induced Exosomal circRNA Promotes Metastasis of Colorectal Cancer via Targeting GEF-H1/RhoA axis. Theranostics 10, 8211-8226. doi:10.7150/ thno.44419

Yang, S.-j., Wang, D.-d., Zhong, S.-l., Chen, W.-q., Wang, F.-l., Zhang, J., et al. (2021b). Tumor-derived Exosomal circPSMA1 Facilitates the Tumorigenesis, Metastasis, and Migration in Triple-Negative Breast Cancer (TNBC) through miR-637/Akt1/B-Catenin (Cyclin D1) axis. Cel Death Dis 12, 420. doi:10.1038/ s41419-021-03680-1

Yang, S., and Li, X. (2018). Recent Advances in Extracellular Vesicles Enriched with Non-coding RNAs Related to Cancers. Genes Dis. 5, 36-42. doi:10.1016/ j.gendis.2017.12.001

Yang, Z.-G., Awan, F. M., Du, W. W., Zeng, Y., Lyu, J., Wu, D., et al. (2017). The Circular RNA Interacts with STAT3, Increasing its Nuclear Translocation and Wound Repair by Modulating Dnmt3a and miR-17 Function. Mol. Ther. 25, 2062-2074. doi:10.1016/j.ymthe.2017.05.022

Yao, X., Mao, Y., Wu, D., Zhu, Y., Lu, J., Huang, Y., et al. (2021). Exosomal Circ_0030167 Derived from BM-MSCs Inhibits the Invasion, Migration, Proliferation and Stemness of Pancreatic Cancer Cells by Sponging miR$338-5 \mathrm{p}$ and Targeting the Wifl/Wnt $8 / \beta$-Catenin axis. Cancer Lett. 512, 38-50. doi:10.1016/j.canlet.2021.04.030

You, X., Vlatkovic, I., Babic, A., Will, T., Epstein, I., Tushev, G., et al. (2015). Neural Circular RNAs Are Derived from Synaptic Genes and Regulated by Development and Plasticity. Nat. Neurosci. 18, 603-610. doi:10.1038/nn.3975

Yu, G., Jung, H., Kang, Y. Y., and Mok, H. (2018). Comparative Evaluation of Celland Serum-Derived Exosomes to Deliver Immune Stimulators to Lymph Nodes. Biomaterials 162, 71-81. doi:10.1016/j.biomaterials.2018.02.003

Zang, J., Lu, D., and Xu, A. (2020). The Interaction of circRNAs and RNA Binding Proteins: An Important Part of circRNA Maintenance and Function. J. Neurosci. Res. 98, 87-97. doi:10.1002/jnr.24356

Zeng, W., Liu, Y., Li, W. T., Li, Y., and Zhu, J. F. (2020). CircFNDC3B Sequestrates miR-937-5p to Derepress TIMP3 and Inhibit Colorectal Cancer Progression. Mol. Oncol. 14, 2960-2984. doi:10.1002/1878-0261.12796

Zeng, Y., Du, W. W., Wu, Y., Yang, Z., Awan, F. M., Li, X., et al. (2017). A Circular RNA Binds to and Activates AKT Phosphorylation and Nuclear Localization Reducing Apoptosis and Enhancing Cardiac Repair. Theranostics 7, 3842-3855. doi:10.7150/thno.19764
Zhan, M., Zhao, H., and Han, Z. C. (2004). Signalling Mechanisms of Anoikis. Histol. Histopathol 19, 973-983. doi:10.14670/HH-19.973

Zhang, C., Cao, J., Lv, W., and Mou, H. (2021). CircRNA_100395 Carried by Exosomes from Adipose-Derived Mesenchymal Stem Cells Inhibits the Malignant Transformation of Non-small Cell Lung Carcinoma through the miR-141-3p-LATS2 Axis. Front. Cel Dev. Biol. 9, 663147. doi:10.3389/ fcell.2021.663147

Zhang, L., and Yu, D. (2019). Exosomes in Cancer Development, Metastasis, and Immunity. Biochim. Biophys. Acta (Bba) - Rev. Cancer 1871, 455-468. doi:10.1016/j.bbcan.2019.04.004

Zhang, N., Nan, A., Chen, L., Li, X., Jia, Y., Qiu, M., et al. (2020). Circular RNA circSATB2 Promotes Progression of Non-small Cell Lung Cancer Cells. Mol. Cancer 19, 101. doi:10.1186/s12943-020-01221-6

Zhang, X.-O., Dong, R., Zhang, Y., Zhang, J.-L., Luo, Z., Zhang, J., et al. (2016). Diverse Alternative Back-Splicing and Alternative Splicing Landscape of Circular RNAs. Genome Res. 26, 1277-1287. doi:10.1101/gr.202895.115

Zhang, X., Wang, S., Wang, H., Cao, J., Huang, X., Chen, Z., et al. (2019). Circular RNA circNRIP1 Acts as a microRNA-149-5p Sponge to Promote Gastric Cancer Progression via the AKT1/mTOR Pathway. Mol. Cancer 18, 20. doi:10.1186/s12943-018-0935-5

Zhao, L., Liu, W., Xiao, J., and Cao, B. (2015). The Role of Exosomes and "Exosomal Shuttle microRNA" in Tumorigenesis and Drug Resistance. Cancer Lett. 356, 339-346. doi:10.1016/j.canlet.2014.10.027

Zhao, R., Chen, X., Song, H., Bie, Q., and Zhang, B. (2020). Dual Role of MSCDerived Exosomes in Tumor Development. Stem Cell Int. 2020, 1-11. doi: $10.1155 / 2020 / 8844730$

Zhong, Y., Du, Y., Yang, X., Mo, Y., Fan, C., Xiong, F., et al. (2018). Circular RNAs Function as ceRNAs to Regulate and Control Human Cancer Progression. Mol. Cancer 17, 79. doi:10.1186/s12943-018-0827-8

Zhou, Y., Yu, Z., Wang, X., Chen, W., Liu, Y., Zhang, Y., et al. (2021). Exosomal circRNAs Contribute to Intestinal Development via the VEGF Signalling Pathway in Human Term and Preterm Colostrum. Aging 13, 11218-11233. doi:10.18632/aging.202806

Zhu, C., Su, Y., Liu, L., Wang, S., Liu, Y., and Wu, J. (2021). Circular RNA Hsa_circ_0004277 Stimulates Malignant Phenotype of Hepatocellular Carcinoma and Epithelial-Mesenchymal Transition of Peripheral Cells. Front. Cel Dev. Biol. 8, 585565. doi:10.3389/fcell.2020.585565

Zhuang, X., Zhang, H., and Hu, G. (2019). Cancer and Microenvironment Plasticity: Double-Edged Swords in Metastasis. Trends Pharmacol. Sci. 40, 419-429. doi:10.1016/j.tips.2019.04.005

Zong, Z.-H., Du, Y.-P., Guan, X., Chen, S., and Zhao, Y. (2019). CircWHSC1 Promotes Ovarian Cancer Progression by Regulating MUC1 and hTERT through Sponging miR-145 and miR-1182. J. Exp. Clin. Cancer Res. 38, 437. doi:10.1186/s13046-019-1437-z

Conflict of Interest: The authors declare that the research was conducted in the absence of any commercial or financial relationships that could be construed as a potential conflict of interest.

Publisher's Note: All claims expressed in this article are solely those of the authors and do not necessarily represent those of their affiliated organizations, or those of the publisher, the editors and the reviewers. Any product that may be evaluated in this article, or claim that may be made by its manufacturer, is not guaranteed or endorsed by the publisher.

Copyright (c) $2021 \mathrm{Zhou}, \mathrm{He}, \mathrm{He}, \mathrm{Ou}$ and Cao. This is an open-access article distributed under the terms of the Creative Commons Attribution License (CC BY). The use, distribution or reproduction in other forums is permitted, provided the original author(s) and the copyright owner(s) are credited and that the original publication in this journal is cited, in accordance with accepted academic practice. No use, distribution or reproduction is permitted which does not comply with these terms. 


\section{GLOSSARY}

AMSC adipose-derived MSC

CAA cholangiocarcinoma

CAFs Cancer-associated fibroblasts

ceRNA competitive endogenous RNA

circRNAs circular RNAs

CRC colorectal cancer

DCs dendritic cells

DFS disease-free survival

ECM extracellular matrix

BM-MSC bone marrow MSC

EMT Epithelial-to-mesenchymal transition

ESCRT endosomal sorting complex required for transport

GC gastric cancer

HCC hepatocellular carcinoma

ILC innate lymphocytes

lncRNAs long non-coding RNAs
MDSCs myeloid-derived suppressor cells

miRNAs microRNAs

MMP2 matrix metallopeptidase 2

MREs miRNA response elements

MSCs Mesenchymal stem cells

MVBs multivesicular bodies

NK cells natural killer cells

NSCLC non-small cell lung cancer

PMN pre-metastatic niche

RBPs RNA binding proteins

ROC receiver operating characteristic

SCLC Small cell lung cancer

TAMs tumor-associated macrophages

TME tumor microenvironment

TNBC triple-negative breast cancer

TNM tumor-lymph node metastasis

UCB urothelial carcinoma of the bladder

UTRs untranslated regions 